Research Article

\title{
Elastodynamic Solutions of a Finite Soil Layer under Interior Distributed Actions
}

\author{
Shiping Zhang $\mathbb{D}^{1,2}$ and Zhan $\mathrm{Xu} \mathbb{D}^{1,2}$ \\ ${ }^{1}$ Key Laboratory of Road Structure and Material of Ministry of Transport (Changsha), \\ Changsha University of Science \& Technology, Changsha 410114, China \\ ${ }^{2}$ School of Traffic and Transportation Engineering, Changsha University of Science \& Technology, Changsha 410114, \\ Hunan, China \\ Correspondence should be addressed to Shiping Zhang; zhangshipingwy@126.com
}

Received 29 January 2021; Accepted 11 June 2021; Published 30 June 2021

Academic Editor: Changjie Zheng

Copyright ( 2021 Shiping Zhang and Zhan Xu. This is an open access article distributed under the Creative Commons Attribution License, which permits unrestricted use, distribution, and reproduction in any medium, provided the original work is properly cited.

\begin{abstract}
Through a method of displacement potentials, Fourier series, and Hankel integral transformation, the generalized solutions of an elastic layer resting on a rigid base under arbitrary, distributed, buried, and time-harmonic loads are developed in this study. With the proposed solution, the specific results for two kinds of uniform distributions as a kind of fundamental solutions in the interaction analysis of media and inclusions by the method of boundary integral equations are included as illustrations. Finally, numerical examples involving surface and buried patch loads are presented to validate the solutions and examine the effects of the thickness of the elastic layer. The results show that the proposed solution can cover the classical half-space solution by taking enough large thickness of the elastic layer (e.g., the ratio of the layer thickness beneath the load to the load radius $\geq 50$ ) and the surface load solution by setting the load depth to zero; the underlying rigid base has significant and complex influence on the dynamic response of the thin layer due to wave reflections, which needs to be considered in the design and practice of related engineering.
\end{abstract}

\section{Introduction}

Mechanical behaviors of soils under dynamic loads, such as traffic loads, construction operations, machinery vibrations, and earthquakes, are of fundamental importance in pavement engineering, geotechnical, and seismic engineering [1-3]. By treating soil materials as single-phase elastic or viscoelastic media, their dynamic responses to external loads have been studied extensively in classical treatments [4-6]. Using the theories and methods developed for single-phase solids, research on the propagation of elastic waves in poroelastic materials also has continued to increase since the pioneering work of Biot [7].

The research began with the surface load cases involving either point, line, or patch loads, which characterize certain kinds of spatial symmetry and simplification. For instance, Lee [8], Yan et al. [9], Jones [10], Liu and Pan [11], Andersen et al. [12], and You et al. [13] studied the dynamic response problems of single-phase elastic layered half-spaces subjected to vertical surface loads. Paul [14, 15], Halpern and Christiano [16], Puswewala and Rajapakse [17], Senjuntichai and Rajapakse [18], Jin and Liu [19], and Feng et al. [20] investigated the axisymmetric responses of poroelastic halfspaces under vertical or radial ring surface loads.

More general and mathematically demanding cases are those when asymmetric sources are buried inside media, which are generally corresponding to the interaction problems of media and embedded structures (e.g., geogrid reinforcement, pile foundation, or buried pipes), blast loads, or seismic loads. For example, Khojasteh et al. [21], Liu et al. [22], Lin et al. [23], Park and Kaynia [24], Noori et al. [25], Ai and Li [26], and Ai et al. [27] analyzed the dynamic responses of single-phase multilayered half-spaces to buried vertical or horizontal loads. 
Philippacopoulos [28], Jin and Liu [29], Zhou et al. [30], Chen et al. [31], Zheng et al. [32, 33], and Pooladi et al. [34] studied the dynamic responses of poroelastic halfspaces due to buried point or distributed loads.

The media in all the foregoing studies are modeled as half-spaces, which are available when their thicknesses are large enough. However, a more common case of many soil systems found in practice is that of finite soil stratum underlain by stiff bedrock. The existence of the bedrock boundary in this physical system leads to dynamic behavior with both resonant and cutoff frequency phenomena in wave mechanics. The significant differences in geometry and boundary conditions of the finite-layered media and the well-known infinite half-space clearly warrant careful attention in design and practice. For the finite-layered medium problems, Zheng [35] studied the dynamic response of an elastic layer on stiff foundation under time-harmonic surface vertical concentrated load. Chen et al. [36] presented a semianalytical solution for the axisymmetric consolidation of a transversely isotropic soil layer resting on a rough impervious base and subjected to a uniform circular pressure at the ground surface. Kim [37] proposed the general viscoelastic solutions for multilayered systems subjected to static and moving loads. Dan et al. [38] performed the coupled hydromechanical response analysis of saturated asphalt pavement under moving traffic load. Recently, Yuan and Wang [39] investigated the effects of surface tension on the two-dimensional contact problems of an elastic layer bonded to the rigid substrate.

These studies, however, were only suitable for surface load cases, while unable to reflect the effect of interior actions. Together with the fundamental importance of buried load solutions in the soil-structure interaction analysis, the expected generality significance that buried load solutions can be reduced to surface load solutions, and finite-layered solutions to half-space solutions. To this end, an analytical investigation for time-harmonic response analysis of an elastic layer resting on rigid base under arbitrary, distributed, and interior excitations is initiated to enhance the current framework for this class of problems. The remainder of this study is structured as follows: In Section 2, displacement potentials are introduced to fully decouple the field equations into three independent wave equations, followed by a finite Fourier transformation and a Hankel transformation, which reduce the dimension of wave equations to one. Then, general solutions to arbitrary, distributed, and buried loads are obtained by imposing the boundary condition and the conditions at the load level. In Section 3, specific responses to buried circular patch loads with uniform distribution are presented as application illustrations of the generalized solution. By degenerating the model to a half-space and comparing it with classical results and comparing the present solution with FEM results, the present solution is validated in Section 4. Also, the effect of the thickness of the elastic layer on its response of displacements is investigated with numerical examples.

\section{Statement of Problem and Its Solutions}

As mentioned earlier, the problem under consideration is that of an arbitrary internal body force field distributed on the plane $z=s$ in a finite stratum of homogeneous, isotropic, and linearly elastic medium resting on rigid base, as shown in Figure 1. In terms of elastodynamics, the equation of motion for the elastic layer can be expressed as

$$
\left(\lambda^{s}+\mu^{s}\right) \nabla\left(\nabla \cdot \mathbf{u}_{s}\right)+\mu^{s} \nabla \cdot\left(\nabla \mathbf{u}_{s}\right)-\rho^{s} \ddot{\mathrm{u}}_{\mathrm{s}}=0,
$$

where $\lambda^{s}$ and $\mu^{s}$ are the two Lame constants of the layer and $\rho^{s}$ represents the density of the layer. $\mathbf{u}_{s}$ is the displacement vector of the layer, and $\ddot{u}_{\mathrm{s}}$ is its acceleration vector. $\nabla$ denotes the gradient operator.

From Figure 1, the buried load source at $z=s$ can be written as

$$
\sigma_{z r}^{s}\left(r, \theta, s^{-}, t\right)-\sigma_{z r}^{s}\left(r, \theta, s^{+}, t\right)= \begin{cases}P(r, \theta, t) & (r, \theta, s) \in \pi_{s} \\ 0 & (r, \theta, s) \notin \pi_{s}\end{cases}
$$

$$
\sigma_{z \theta}^{s}\left(r, \theta, s^{-}, t\right)-\sigma_{z \theta}^{s}\left(r, \theta, s^{+}, t\right)=\left\{\begin{array}{ll}
Q(r, \theta, t) & (r, \theta, s) \in \pi_{s} \\
0 & (r, \theta, s) \notin \pi_{s}
\end{array},\right.
$$

$$
\sigma_{z z}^{s}\left(r, \theta, s^{-}, t\right)-\sigma_{z z}^{s}\left(r, \theta, s^{+}, t\right)=\left\{\begin{array}{ll}
R(r, \theta, t) & (r, \theta, s) \in \pi_{s} \\
0 & (r, \theta, s) \notin \pi_{s}
\end{array}{ }^{\prime},\right.
$$

where $P, Q$, and $R$ represent the stress distributions in directions $r, \theta$, and $z$, respectively, $\sigma_{z r}^{s}, \sigma_{z \theta}^{s}$, and $\sigma_{z z}^{s}$ are stress components, and $\pi_{s}$ is the distribution area of applied loads.

Here it is assumed that the surface of the layer is freetraction and its bottom is bonded on the base, i.e.,

$$
\begin{gathered}
\sigma_{z r}^{s}(r, \theta, 0, t)=\sigma_{z \theta}^{s}(r, \theta, 0, t)=\sigma_{z z}^{s}(r, \theta, 0, t)=0, \\
u_{s}(r, \theta, L, t)=v_{s}(r, \theta, L, t)=w_{s}(r, \theta, L, t)=0,
\end{gathered}
$$

and all displacements at $z=s$ are continuous, where $u_{s}, v_{s}$, and $w_{s}$ are displacement components.

Since time-harmonic loads with the time term $e^{i \omega t}$ are considered in this paper, field variables can be written as

$$
\begin{aligned}
\sigma_{z r}^{s}(r, \theta, z, t) & =\sigma_{z r}^{s}(r, \theta, z) e^{i \omega t}, \\
\sigma_{z \theta}^{s}(r, \theta, z, t) & =\sigma_{z \theta}^{s}(r, \theta, z) e^{i \omega t}, \\
\sigma_{z z}^{s}(r, \theta, z, t) & =\sigma_{z z}^{s}(r, \theta, z) e^{i \omega t}, \\
u_{s}(r, \theta, z, t) & =u_{s}(r, \theta, z) e^{i \omega t}, \\
v_{s}(r, \theta, z, t) & =v_{s}(r, \theta, z) e^{i \omega t}, \\
w_{s}(r, \theta, z, t) & =w_{s}(r, \theta, z) e^{i \omega t} .
\end{aligned}
$$

For brevity, the time term $e^{i \omega t}$ is suppressed in the following analyses. Therefore, equation (1) can be represented as 


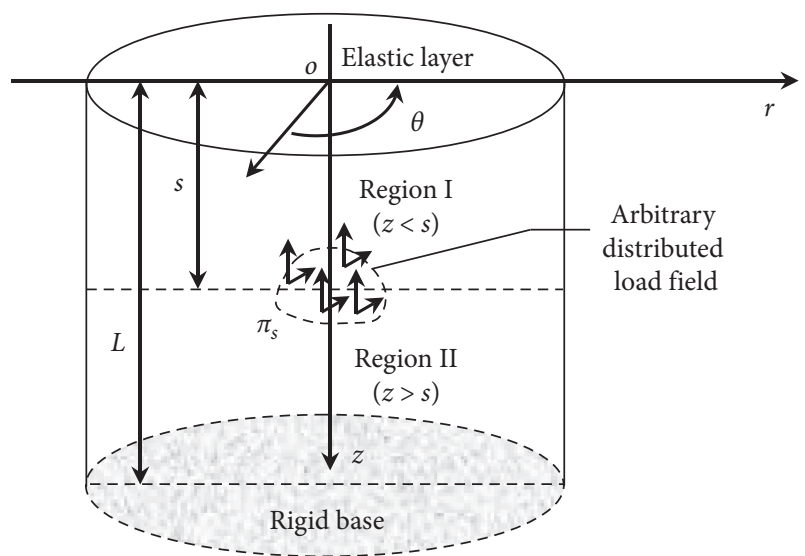

Figure 1: An elastic layer under buried, arbitrary, distributed, and time-harmonic loads.

$$
\left(\lambda^{s}+\mu^{s}\right) \nabla\left(\nabla \cdot \mathbf{u}_{s}\right)+\mu^{s} \nabla \cdot\left(\nabla \mathbf{u}_{s}\right)+\rho^{s} \omega^{2} \mathbf{u}_{s}=0 .
$$

Important to our further development is the resolution of displacement field by three scalar potentials inspired by Pak [6], i.e.,

$$
\mathbf{u}_{s}(r, \theta, z)=\nabla \phi_{s}(r, \theta, z)+\nabla \times\left(\chi_{s}(r, \theta, z) \mathbf{e}_{z}+\nabla \times\left(\eta_{s}(r, \theta, z) \mathbf{e}_{z}\right)\right),
$$

where $\mathbf{e}_{z}$ is the unit vector along the $z$-direction of the cylindrical coordinate system.

Then, the component form of equation (6) is further written as

$$
\begin{aligned}
& u_{s}=\frac{\partial \phi_{s}}{\partial r}+\frac{\partial \chi_{s}}{r \partial \theta}+\frac{\partial^{2} \eta_{s}}{\partial r \partial z} \\
& v_{s}=\frac{\partial \phi_{s}}{r \partial \theta}+\frac{\partial^{2} \eta_{s}}{r \partial \theta \partial z}-\frac{\partial \chi_{s}}{\partial r}, \\
& w_{s}=\frac{\partial \phi_{s}}{\partial z}-\left(\frac{\partial^{2} \eta_{s}}{\partial r^{2}}+\frac{\partial^{2} \eta_{s}}{r^{2} \partial \theta^{2}}+\frac{\partial \eta_{s}}{r \partial r}\right) .
\end{aligned}
$$

By the imposition of equation (6), the equations of motion in terms of potential functions can be obtained from equation (5) as

$$
\begin{aligned}
& \nabla^{2} \phi_{s}(r, \theta, z)+k_{1}^{2} \phi_{s}(r, \theta, z)=0, \\
& \nabla^{2} \chi_{s}(r, \theta, z)+k_{2}^{2} \chi_{s}(r, \theta, z)=0, \\
& \nabla^{2} \eta_{s}(r, \theta, z)+k_{2}^{2} \eta_{s}(r, \theta, z)=0,
\end{aligned}
$$

where $k_{1}^{2}=\omega^{2} / V_{d}^{2}$ and $k_{2}^{2}=\omega^{2} / V_{s}^{2} . V_{d}=\sqrt{\lambda^{s}+2 \mu^{s} / \rho^{s}}$ denotes the speed of the dilatational wave, and $V_{s}=\sqrt{\mu^{s} / \rho^{s}}$ is the speed of the shearing wave. $\nabla^{2}=\left(\partial^{2} / \partial r^{2}\right)+(\partial / r \partial r)+$ $\left(\partial^{2} / r^{2} \partial \theta^{2}\right)+\left(\partial^{2} / \partial z^{2}\right)$ is the Laplacian operator.

Next integral transformations are introduced to solve equation (8). First, by a Fourier expansion with respect to $\theta$, these potential functions can be written as

$$
\begin{aligned}
& \phi_{s}(r, \theta, z)=\sum_{n=-\infty}^{+\infty} \phi_{s n}(r, z) e^{\mathrm{in} \theta}, \\
& \chi_{s}(r, \theta, z)=\sum_{n=-\infty}^{+\infty} \chi_{s n}(r, z) e^{\mathrm{in} \theta}, \\
& \eta_{s}(r, \theta, z)=\sum_{n=-\infty}^{+\infty} \eta_{s n}(r, z) e^{\mathrm{in} \theta} .
\end{aligned}
$$

The displacement components can be written as

$$
\begin{aligned}
& u_{s}(r, \theta, z)=\sum_{n=-\infty}^{+\infty} u_{s n}(r, z) e^{\mathrm{in} \theta}, \\
& v_{s}(r, \theta, z)=\sum_{n=-\infty}^{+\infty} v_{s n}(r, z) e^{\mathrm{in} \theta}, \\
& w_{s}(r, \theta, z)=\sum_{n=-\infty}^{+\infty} w_{s n}(r, z) e^{\mathrm{in} \theta}
\end{aligned}
$$

and the source distributions in equations $(2 a)-(2 c)$ can be written as

$$
\begin{aligned}
& P(r, \theta, z)=\sum_{n=-\infty}^{+\infty} P_{n}(r, z) e^{\mathrm{in} \theta}, \\
& Q(r, \theta, z)=\sum_{n=-\infty}^{+\infty} Q_{n}(r, z) e^{\mathrm{in} \theta}, \\
& R(r, \theta, z)=\sum_{n=-\infty}^{+\infty} R_{n}(r, z) e^{\mathrm{in} \theta}
\end{aligned}
$$

The equations for the Fourier series coefficients of the potentials can then be derived from the three wave equations (8), i.e.,

$$
\begin{aligned}
& \left(\frac{\partial^{2}}{\partial r^{2}}+\frac{\partial}{r \partial r}-\frac{n^{2}}{r^{2}}+\frac{\partial^{2}}{\partial z^{2}}\right) \phi_{s n}(r, z)+k_{1}^{2} \phi_{s n}(r, z)=0, \\
& \left(\frac{\partial^{2}}{\partial r^{2}}+\frac{\partial}{r \partial r}-\frac{n^{2}}{r^{2}}+\frac{\partial^{2}}{\partial z^{2}}\right) \chi_{s n}(r, z)+k_{2}^{2} \chi_{s n}(r, z)=0, \\
& \left(\frac{\partial^{2}}{\partial r^{2}}+\frac{\partial}{r \partial r}-\frac{n^{2}}{r^{2}}+\frac{\partial^{2}}{\partial z^{2}}\right) \eta_{s n}(r, z)+k_{2}^{2} \eta_{s n}(r, z)=0 .
\end{aligned}
$$

Then, applying the Hankel transformation,

$$
\tilde{f}^{n}(\xi)=\int_{0}^{\infty} r f(r) J_{n}(\xi r) \mathrm{d} r
$$

whose inverse transformation is

$$
f(r)=\int_{0}^{\infty} \xi \widetilde{f}^{n}(\xi) J_{n}(\xi r) \mathrm{d} \xi
$$

to equation (12) yields 


$$
\begin{aligned}
& \frac{\partial^{2}}{\partial z^{2}} \widetilde{\phi}_{s n}^{n}(\xi, z)-\left(\xi^{2}-k_{1}^{2}\right) \widetilde{\phi}_{s n}^{n}(\xi, z)=0, \\
& \frac{\partial^{2}}{\partial z^{2}} \widetilde{\chi}_{s n}^{n}(\xi, z)-\left(\xi^{2}-k_{2}^{2}\right) \widetilde{\chi}_{s n}^{n}(\xi, z)=0, \\
& \frac{\partial^{2}}{\partial z^{2}} \widetilde{\eta}_{s n}^{n}(\xi, z)-\left(\xi^{2}-k_{2}^{2}\right) \widetilde{\eta}_{s n}^{n}(\xi, z)=0 .
\end{aligned}
$$

The general solutions of equation (15) can be easily found to be

$$
\begin{aligned}
& \widetilde{\phi}_{s n}^{n}(\xi, z)=A_{n}^{I} e^{\alpha z}+B_{n}^{I} e^{-\alpha z}, \\
& \widetilde{\chi}_{s n}^{n}(\xi, z)=C_{n}^{I} e^{\beta z}+D_{n}^{I} e^{-\beta z}, \\
& \widetilde{\eta}_{s n}^{n}(\xi, z)=E_{n}^{I} e^{\beta z}+F_{n}^{I} e^{-\beta z}, \quad \text { in region } I,
\end{aligned}
$$

and

$$
\begin{aligned}
& \widetilde{\phi}_{s n}^{n}(\xi, z)=A_{n}^{I I} e^{\alpha z}+B_{n}^{I I} e^{-\alpha z}, \\
& \tilde{\chi}_{s n}^{n}(\xi, z)=C_{n}^{I I} e^{\beta z}+D_{n}^{I I} e^{-\beta z}, \\
& \widetilde{\eta}_{s n}^{n}(\xi, z)=E_{n}^{I I} e^{\beta z}+F_{n}^{I I} e^{-\beta z}, \quad \text { in region } I I,
\end{aligned}
$$

where $\alpha=\left(\xi^{2}-k_{1}^{2}\right)^{1 / 2}, \quad \beta=\left(\xi^{2}-k_{2}^{2}\right)^{1 / 2}, \quad$ and $\quad A_{n}^{I} \sim F_{n}^{I}$, $A_{n}^{I I} \sim F_{n}^{I I}$ are unknown coefficients of integration which are determined by the interfacial and boundary conditions. The branch cut of the radicals $\alpha$ and $\beta$ is specified such that $\operatorname{Re}(\alpha) \geq 0$ and $\operatorname{Re}(\beta) \geq 0$.

Now, it is noted that the free surface and bottom conditions provide 6 equations (equation (3)); the stress discontinuity across the region interface provides 3 equations (equations $(2 \mathrm{a})-(2 \mathrm{c})$ ); and the continuity of soil displacements provides the other 3 equations.

For further usage, the displacement-potential relationship in the cylindrical coordinate systems and integral transformation domains obtained by substituting equations (9), (10), and (13) into equation (7) are given below:

$$
\begin{gathered}
\widetilde{u}_{s n}^{n+1}(\xi, z)+i \widetilde{v}_{s n}^{n+1}(\xi, z)=-\xi \widetilde{\phi}_{s n}^{n}(\xi, z)+i \xi \widetilde{\chi}_{s n}^{n}(\xi, z)-\xi \frac{\partial}{\partial z} \widetilde{\eta}_{s n}^{n}(\xi, z), \\
\widetilde{u}_{s n}^{n-1}(\xi, z)+i \widetilde{v}_{s n}^{n-1}(\xi, z)=-\xi \widetilde{\phi}_{s n}^{n}(\xi, z)+i \xi \widetilde{\chi}_{s n}^{n}(\xi, z)+\xi \frac{\partial}{\partial z} \widetilde{\eta}_{s n}^{n}(\xi, z), \\
\widetilde{w}_{s n}^{n}(\xi, z)=\frac{\partial}{\partial z} \widetilde{\phi}_{s n}^{n}(\xi, z)+\xi^{2} \widetilde{\eta}_{s n}^{n}(\xi, z),
\end{gathered}
$$

and the stress-potential relationship derived by using Hook's law and equations (7), (9), (10) and (13) can be expressed as

$$
\begin{gathered}
\tilde{\sigma}_{z z n}^{s n}(\xi, z)=\left[2 \mu^{s} \frac{\partial^{2}}{\partial z^{2}} \widetilde{\phi}_{s n}^{n}(\xi, z)-\lambda^{s} k_{1}^{2} \widetilde{\phi}_{s n}^{n}(\xi, z)\right]+2 \mu^{s} \xi^{2} \frac{\partial}{\partial z} \widetilde{\eta}_{s n}^{n}(\xi, z) \\
\widetilde{\sigma}_{z r n}^{s(n+1)}(\xi, z)+i \widetilde{\sigma}_{z \theta n}^{s(n+1)}(\xi, z)=-2 \mu^{s} \xi \frac{\partial}{\partial z} \widetilde{\phi}_{s n}^{n}(\xi, z)+i \mu^{s} \xi \frac{\partial}{\partial z} \widetilde{\chi}_{s n}^{n}(\xi, z)-\mu^{s} \xi\left[\frac{\partial^{2}}{\partial z^{2}} \widetilde{\eta}_{s n}^{n}(\xi, z)+\xi^{2} \widetilde{\eta}_{s n}^{n}(\xi, z)\right] \\
\widetilde{\sigma}_{z r n}^{s(n-1)}(\xi, z)-i \widetilde{\sigma}_{z \theta n}^{s(n-1)}(\xi, z)=2 \mu^{s} \xi \frac{\partial}{\partial z} \widetilde{\phi}_{s n}^{n}(\xi, z)+i \mu^{s} \xi \frac{\partial}{\partial z} \widetilde{\chi}_{s n}^{n}(\xi, z)+\mu^{s} \xi\left[\frac{\partial^{2}}{\partial z^{2}} \widetilde{\eta}_{s n}^{n}(\xi, z)+\xi^{2} \widetilde{\eta}_{s n}^{n}(\xi, z)\right]
\end{gathered}
$$

After the determination of the unknown coefficients by the set of boundary condition equations, one can obtain the solutions of field variables in forms 


$$
\begin{aligned}
\widetilde{u}_{s n}^{n+1}(\xi, z)+i \widetilde{v}_{s n}^{n+1}(\xi, z) & =\gamma_{1} \frac{X_{n}-Y_{n}}{2 \mu^{s}}+\gamma_{2} \frac{Y_{n}+X_{n}}{2 \mu^{s}}-\gamma_{3} \frac{Z_{n}}{\left(\lambda^{s}+2 \mu^{s}\right)}, \\
\widetilde{u}_{s n}^{n-1}(\xi, z)-i \widetilde{v}_{s n}^{n-1}(\xi, z) & =-\gamma_{1} \frac{X_{n}-Y_{n}}{2 \mu^{s}}+\gamma_{2} \frac{Y_{n}+X_{n}}{2 \mu^{s}}+\gamma_{3} \frac{Z_{n}}{\left(\lambda^{s}+2 \mu^{s}\right)}, \\
\widetilde{w}_{s n}^{n}(\xi, z) & =-\gamma_{4} \frac{X_{n}-Y_{n}}{2 \mu^{s}}+\gamma_{5} \frac{Z_{n}}{\left(\lambda^{s}+2 \mu^{s}\right)},
\end{aligned}
$$

and

$$
\begin{gathered}
\widetilde{\sigma}_{z z n}^{s n}(\xi, z)=-\gamma_{6} \frac{X_{n}+Y_{n}}{2 \mu^{s}}+\gamma_{7} \frac{Z_{n}}{\left(\lambda^{s}+2 \mu^{s}\right)}, \\
\widetilde{\sigma}_{z r n}^{s(n+1)}(\xi, z)+i \widetilde{\sigma}_{z \theta n}^{s(n+1)}(\xi, z)=\gamma_{8} \frac{X_{n}-Y_{n}}{2}+\gamma_{9} \frac{Y_{n}+X_{n}}{2}-\gamma_{10} \frac{Z_{n}}{\left(\lambda^{s}+2 \mu^{s}\right)}, \\
\widetilde{\sigma}_{z r n}^{s(n-1)}(\xi, z)-i \widetilde{\sigma}_{z \theta n}^{s(n-1)}(\xi, z)=-\gamma_{8} \frac{X_{n}-Y_{n}}{2} \gamma_{9} \frac{Y_{n}+X_{n}}{2}+\frac{Z_{n}}{\left(\lambda^{s}+2 \mu^{s}\right)},
\end{gathered}
$$

where

$$
\gamma_{i}=\left\{\begin{array}{ll}
\gamma_{i}^{-} & z<s \\
\gamma_{i}^{+} & z>s
\end{array}, \quad i=1 \sim 10,\right.
$$

and the coefficients $\gamma_{i}^{-}, \gamma_{i}^{+}, X_{n}, Y_{n}$, and $Z_{n}$ are provided in Appendix.

By applying the inverse Hankel transformation to the solutions in equations (19) and (20) and then inserting the inverted Fourier components into the Fourier series expansion in equations (9)-(11), the time-harmonic response of the layer to the general buried loads is formally developed below.

$$
\begin{aligned}
u_{s}(r, \theta, z, t)= & \frac{1}{4 \mu^{\mu}} e^{i \omega t} \sum_{n=-\infty}^{+\infty}\left\{e^{\operatorname{in} \theta} \int_{0}^{\infty} \xi \gamma_{1}\left(X_{n}-Y_{n}\right)\left[J_{n+1}(\xi r)-J_{n-1}(\xi r)\right] \mathrm{d} \xi\right\} \\
& +\frac{1}{4 \mu^{s}} e^{i \omega t} \sum_{n=-\infty}^{+\infty}\left\{e^{\operatorname{in} \theta} \int_{0}^{\infty} \xi \gamma_{2}\left(Y_{n}+X_{n}\right)\left[J_{n+1}(\xi r)+J_{n-1}(\xi r)\right] \mathrm{d} \xi\right\} \\
& -\frac{1}{2\left(\lambda^{s}+2 \mu^{s}\right)} e^{i \omega t} \sum_{n=-\infty}^{+\infty}\left\{e^{\operatorname{in} \theta} \int_{0}^{\infty} \xi \gamma_{3} Z_{n}\left[J_{n+1}(\xi r)-J_{n-1}(\xi r)\right] \mathrm{d} \xi\right\}, \\
v_{s}(r, \theta, z, t)= & \frac{1}{4 i \mu^{s}} e^{i \omega t} \sum_{n=-\infty}^{+\infty}\left\{e^{\operatorname{in} \theta} \int_{0}^{\infty} \xi \gamma_{1}\left(X_{n}-Y_{n}\right)\left[J_{n+1}(\xi r)+J_{n-1}(\xi r)\right] \mathrm{d} \xi\right\} \\
& +\frac{1}{4 i \mu^{s}} e^{i \omega t} \sum_{n=-\infty}^{+\infty}\left\{e^{\operatorname{in} \theta} \int_{0}^{\infty} \xi \gamma_{2}\left(Y_{n}+X_{n}\right)\left[J_{n+1}(\xi r)-J_{n-1}(\xi r)\right] \mathrm{d} \xi\right\} \\
& -\frac{1}{2 i\left(\lambda^{s}+2 \mu^{s}\right)} e^{i \omega t} \sum_{n=-\infty}^{+\infty}\left\{e^{\operatorname{in} \theta} \int_{0}^{\infty} \xi \gamma_{3} Z_{n}\left[J_{n+1}(\xi r)+J_{n-1}(\xi r)\right] \mathrm{d} \xi\right\}, \\
w_{s}(r, \theta, z, t)= & -\frac{1}{2 \mu^{s} e^{i \omega t}} \sum_{n=-\infty}^{+\infty}\left\{e^{\operatorname{in} \theta} \int_{0}^{\infty} \xi \gamma_{4}\left(X_{n}-Y_{n}\right) J_{n}(\xi r) \mathrm{d} \xi\right\} \\
& +\frac{1}{\left(\lambda^{s}+2 \mu^{s}\right)} e^{i \omega t} \sum_{n=-\infty}^{+\infty}\left\{e^{\operatorname{in} \theta} \int_{0}^{\infty} \xi \gamma_{5} Z_{n} J_{n}(\xi r) \mathrm{d} \xi\right\}
\end{aligned}
$$




$$
\begin{aligned}
& \sigma_{z r}^{s}(r, \theta, z, t)=-\frac{1}{4} e^{i \omega t} \sum_{n=-\infty}^{+\infty}\left\{e^{\mathrm{in} \theta} \int_{0}^{\infty} \xi \gamma_{8}\left(X_{n}-Y_{n}\right)\left[J_{(n-1)}(\xi r)-J_{(n+1)}(\xi r)\right] \mathrm{d} \xi\right\} \\
& +\frac{1}{4} e^{i \omega t} \sum_{n=-\infty}^{+\infty}\left\{e^{i n \theta} \int_{0}^{\infty} \xi \gamma_{9}\left(Y_{n}+X_{n}\right)\left[J_{(n-1)}(\xi r)+J_{(n+1)}(\xi r)\right] \mathrm{d} \xi\right\} \\
& +\frac{1}{2\left(\lambda^{s}+2 \mu^{s}\right)} e^{i \omega t} \sum_{n=-\infty}^{+\infty}\left\{e^{i n \theta} \int_{0}^{\infty} \xi \gamma_{10} Z_{n}\left[J_{(n-1)}(\xi r)-J_{(n+1)}(\xi r)\right] \mathrm{d} \xi\right\} \\
& \sigma_{z \theta}^{s}(r, \theta, z, t)=\frac{1}{4 i} e^{i \omega t} \sum_{n=-\infty}^{+\infty}\left\{e^{\mathrm{in} \theta} \int_{0}^{\infty} \xi \gamma_{8}\left(X_{n}-Y_{n}\right)\left[J_{(n+1)}(\xi r)+J_{(n-1)}(\xi r)\right] \mathrm{d} \xi\right\} \\
& +\frac{1}{4 i} e^{i \omega t} \sum_{n=-\infty}^{+\infty}\left\{e^{\operatorname{in} \theta} \int_{0}^{\infty} \xi \gamma_{9}\left(Y_{n}+X_{n}\right)\left[J_{(n+1)}(\xi r)-J_{(n-1)}(\xi r)\right] \mathrm{d} \xi\right\} \\
& -\frac{1}{2 i\left(\lambda^{s}+2 \mu^{s}\right)} e^{i \omega t} \sum_{n=-\infty}^{+\infty}\left\{e^{\mathrm{in} \theta} \int_{0}^{\infty} \xi \gamma_{10} Z_{n}\left[J_{(n+1)}(\xi r)+J_{(n-1)}(\xi r)\right] \mathrm{d} \xi\right\}, \\
& \sigma_{z z}^{s}(r, \theta, z, t)=-\frac{1}{2 \mu^{s}} e^{i \omega t} \sum_{n=-\infty}^{+\infty}\left\{e^{\mathrm{in} \theta} \int_{0}^{\infty} \xi \gamma_{6}\left(X_{n}-Y_{n}\right) J_{n}(\xi r) \mathrm{d} \xi\right\} \\
& +\frac{1}{\left(\lambda^{s}+2 \mu^{s}\right)} e^{i \omega t} \sum_{n=-\infty}^{+\infty}\left\{e^{\mathrm{in} \theta} \int_{0}^{\infty} \xi \gamma_{7} Z_{n} J_{n}(\xi r) \mathrm{d} \xi\right\} .
\end{aligned}
$$

\section{Green's Functions of the Elastic Layer under Specific Loads}

In this section, two kinds of special loads are considered to introduce the application of the obtained equations (22a)-(22f).

3.1. Buried Vertical Uniformly Distributed Circular Patch Load. Such a load can be expressed as

$$
R(r, \theta)=R_{0}(r)= \begin{cases}\frac{1}{\pi r_{0}^{2}}, & r<r_{0}, \\ 0, & r \geq r_{0},\end{cases}
$$

and $R_{n}(r)=0$ for $n \neq 0$, and $P_{n}(r)=0, Q_{n}(r)=0$ for all $n$.

Combining equations (23) and (22a)-(22f), one obtains

$$
\begin{aligned}
u_{s}(r, \theta, z) & =-\frac{1}{\left(\lambda^{s}+2 \mu^{s}\right) \pi r_{0}} \int_{0}^{\infty} \gamma_{3}(\xi, z ; s) J_{1}\left(\xi r_{0}\right) J_{1}(\xi r) \mathrm{d} \xi \\
v_{s}(r, \theta, z) & =0 \\
w_{s}(r, \theta, z) & =\frac{1}{\left(\lambda^{s}+2 \mu^{s}\right) \pi r_{0}} \int_{0}^{\infty} \gamma_{5}(\xi, z, s) J_{1}\left(\xi r_{0}\right) J_{0}(\xi r) \mathrm{d} \xi, \\
\sigma_{z r}^{s}(r, \theta, z) & =-\frac{1}{\left(\lambda^{s}+2 \mu^{s}\right) \pi r_{0}} \int_{0}^{\infty} \gamma_{10}(\xi, z ; s) J_{1}\left(\xi r_{0}\right) J_{1}(\xi r) \mathrm{d} \xi
\end{aligned}
$$

$$
\begin{aligned}
& \sigma_{z \theta}^{s}(r, \theta, z)=0 \\
& \sigma_{z z}^{s}(r, \theta, z)=\frac{1}{\left(\lambda^{s}+2 \mu^{s}\right) \pi r_{0}} \int_{0}^{\infty} \gamma_{7}(\xi, z, s) J_{1}\left(\xi r_{0}\right) J_{0}(\xi r) \mathrm{d} \xi
\end{aligned}
$$

\subsection{Buried Horizontal Uniformly Distributed Circular Patch} Load. Such a load can be written as

$$
P(r, \theta)= \begin{cases}\frac{1}{\pi r_{0}^{2}} \cos (\theta), & r<r_{0}, \\ 0, & r \geq r_{0}\end{cases}
$$

$$
Q(r, \theta)= \begin{cases}-\frac{1}{\pi r_{0}^{2}} \sin (\theta), & r<r_{0}, \\ 0, & r \geq r_{0},\end{cases}
$$

such that

$$
P_{1}(r)=P_{-1}(r)=\frac{1}{2 \pi r_{0}^{2}},
$$

and $P_{n}(r)=0$ for $n \neq \pm 1$.

$$
Q_{1}(r)=-Q_{-1}(r)=i \frac{1}{2 \pi r_{0}^{2}},
$$

and $Q_{n}(r)=0$ for $n \neq \pm 1$, and $R_{n}(r)=0$ for all $n$.

Combining equations (26a) and (26b) and (22a)-(22f), one gets 


$$
\begin{aligned}
& u_{s}(r, \theta, z)=\frac{\cos (\theta)}{2 \mu^{s} \pi r_{0}}\left\{\begin{array}{l}
-\int_{0}^{\infty} \gamma_{1}(\xi, z ; s) J_{1}\left(\xi r_{0}\right)\left[J_{0}(\xi r)-J_{2}(\xi r)\right] \mathrm{d} \xi \\
+\int_{0}^{\infty} \gamma_{2}(\xi, z ; s) J_{1}\left(\xi r_{0}\right)\left[J_{0}(\xi r)+J_{2}(\xi r)\right] \mathrm{d} \xi
\end{array}\right\} \\
& v_{s}(r, \theta, z)=-\frac{\sin (\theta)}{2 \mu^{s} \pi r_{0}}\left\{\begin{array}{l}
-\int_{0}^{\infty} \gamma_{1}(\xi, z ; s) J_{1}\left(\xi r_{0}\right)\left[J_{0}(\xi r)+J_{2}(\xi r)\right] \mathrm{d} \xi \\
+\int_{0}^{\infty} \gamma_{2}(\xi, z ; s) J_{1}\left(\xi r_{0}\right)\left[J_{0}(\xi r)-J_{2}(\xi r)\right] \mathrm{d} \xi
\end{array}\right\}, \\
& w_{s}(r, \theta, z)=-\frac{\cos (\theta)}{\mu^{s} \pi r_{0}} \int_{0}^{\infty} \gamma_{4}(\xi, z, s) J_{1}\left(\xi r_{0}\right) J_{1}(\xi r) \mathrm{d} \xi, \\
& \sigma_{z r}^{s}(r, \theta, z)=\frac{\cos (\theta)}{2 \pi r_{0}}\left\{\begin{array}{l}
-\int_{0}^{\infty} \gamma_{8}(\xi, z ; s) J_{1}\left(\xi r_{0}\right)\left[J_{0}(\xi r)-J_{2}(\xi r)\right] \mathrm{d} \xi \\
+\int_{0}^{\infty} \gamma_{9}(\xi, z ; s) J_{1}\left(\xi r_{0}\right)\left[J_{0}(\xi r)+J_{2}(\xi r)\right] \mathrm{d} \xi
\end{array}\right\} \\
& \sigma_{z \theta}^{s}(r, \theta, z)=\frac{\sin (\theta)}{2 \pi r_{0}}\left\{\begin{array}{l}
-\int_{0}^{\infty} \gamma_{8}(\xi, z ; s) J_{1}\left(\xi r_{0}\right)\left[J_{0}(\xi r)-J_{2}(\xi r)\right] \mathrm{d} \xi \\
+\int_{0}^{\infty} \gamma_{9}(\xi, z ; s) J_{1}\left(\xi r_{0}\right)\left[J_{0}(\xi r)+J_{2}(\xi r)\right] \mathrm{d} \xi
\end{array}\right\}, \\
& \sigma_{z z}^{s}(r, \theta, z)=-\frac{\cos (\theta)}{\mu^{s} \pi r_{0}} \int_{0}^{\infty} \gamma_{6}(\xi, z, s) J_{1}\left(\xi r_{0}\right) J_{1}(\xi r) \mathrm{d} \xi .
\end{aligned}
$$

In a similar way, Green's functions of the elastic layer to point loads or ring loads can also be obtained from equations (22a)-(22f).

\section{Reduction of the Solutions and Numerical Examples}

When the layer thickness tends to infinity, it is natural to expect that the present problem should be reduced to a halfspace case. Thus, by setting $L \longrightarrow \infty$, one can derive from equation (19)

$$
\begin{gathered}
\widetilde{u}_{s n}^{n+1}(\xi, z)+i \widetilde{v}_{s n}^{n+1}(\xi, z)=-\bar{\gamma}_{1} \frac{X_{n}-Y_{n}}{2 \mu^{s}}+\bar{\gamma}_{2} \frac{Y_{n}+X_{n}}{2 \mu^{s}}-\bar{\gamma}_{3} \frac{Z_{n}}{\left(\lambda^{s}+2 \mu^{s}\right)}, \\
\widetilde{u}_{s n}^{n-1}(\xi, z)-i \widetilde{v}_{s n}^{n-1}(\xi, z)=\bar{\gamma}_{1} \frac{X_{n}-Y_{n}}{2 \mu^{s}}+\bar{\gamma}_{2} \frac{Y_{n}+X_{n}}{2 \mu^{s}}+\bar{\gamma}_{3} \frac{Z_{n}}{\left(\lambda^{s}+2 \mu^{s}\right)}, \\
\widetilde{w}_{s n}^{n}(\xi, z)=\bar{\gamma}_{4} \frac{X_{n}-Y_{n}}{2 \mu^{s}}+\bar{\gamma}_{5} \frac{Z_{n}}{\left(\lambda^{s}+2 \mu^{s}\right)},
\end{gathered}
$$

where 


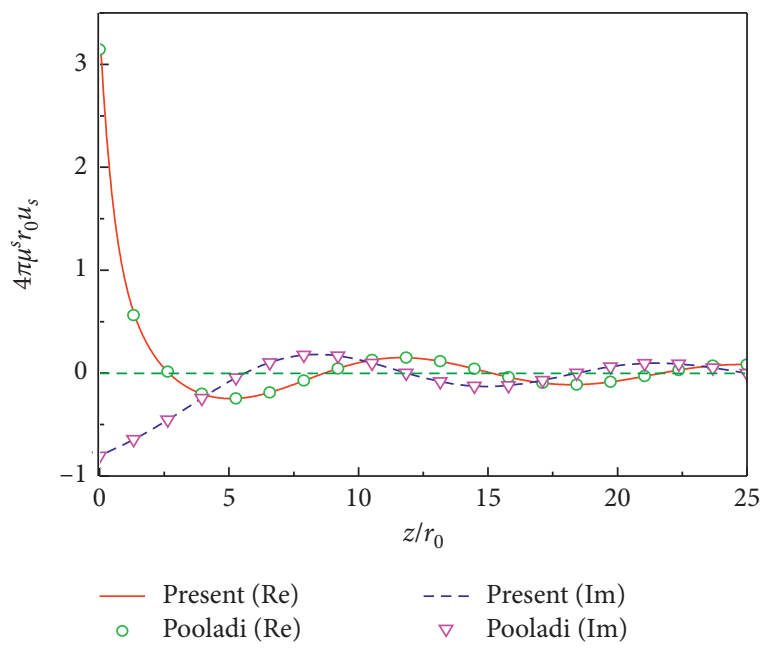

(a)

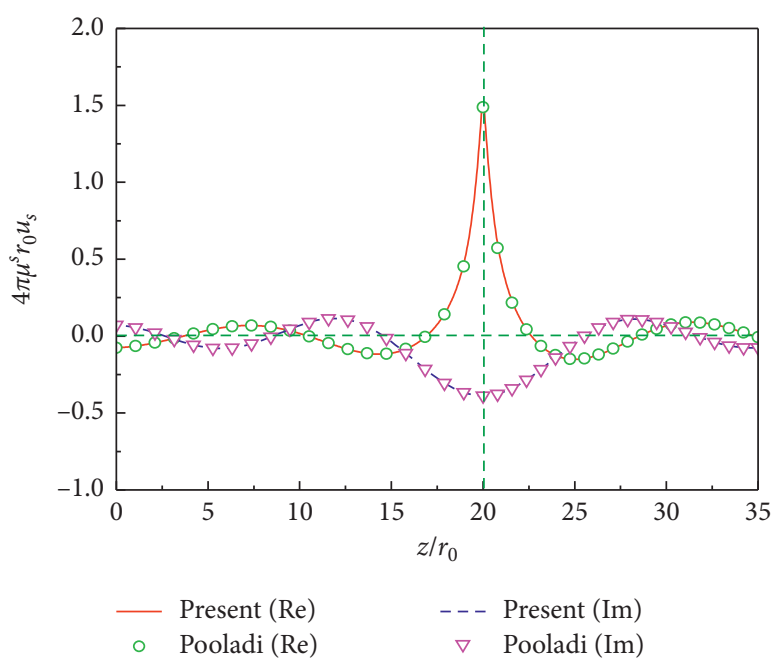

(b)

Figure 2: Lateral displacement $u_{s}$ induced by unit lateral load on $\pi_{s}\left(\bar{\omega}=\omega r_{0} / \sqrt{\mu^{s} / \rho^{s}}=0.5\right)$ : (a) $s=0$; (b) $s=20 r_{0}$.

$$
\begin{aligned}
& \bar{\gamma}_{1}=\frac{\xi^{2}}{2 \alpha k_{2}^{2}} e^{-\alpha|z-s|}-\frac{\beta}{2 k_{2}^{2}} e^{-\beta|z-s|}-\frac{1}{2 k_{2}^{2}} \frac{a_{1}}{a_{2}}\left[\frac{\xi^{2}}{\alpha} e^{-\alpha(s+z)}+\beta e^{-\beta(s+z)}\right]+\frac{2 \mu^{s}\left(2 \xi^{2}-k_{2}^{2}\right) \beta \xi^{2}}{a_{2} k_{2}^{2}}\left[e^{-\alpha s-\beta z}+e^{-\beta s-\alpha z}\right], \\
& \bar{\gamma}_{2}=\frac{1}{2 \beta}\left[e^{-\beta|z-s|}+e^{-\beta(s+z)}\right], \\
& \bar{\gamma}_{3}=\operatorname{sgn}(z, s) \frac{\xi}{2 k_{1}^{2}}\left[e^{-\alpha|z-s|}-e^{-\beta|z-s|}\right]+\frac{\xi}{2 k_{1}^{2}} \frac{a_{1}}{a_{2}}\left[e^{-\alpha(s+z)}+e^{-\beta(s+z)}\right]-\frac{2 \mu^{s}\left(2 \xi^{2}-k_{2}^{2}\right) \xi}{a_{2} k_{1}^{2}}\left[\alpha \beta e^{-\alpha s-\beta z}+\xi^{2} e^{-\beta s-\alpha z}\right], \\
& \bar{\gamma}_{4}=-\operatorname{sgn}(z, s) \frac{\xi}{2 k_{2}^{2}}\left[e^{-\alpha|z-s|}-e^{-\beta|z-s|}\right]+\frac{\xi}{2 k_{2}^{2}} \frac{a_{1}}{a_{2}}\left[e^{-\alpha(s+z)}+e^{-\beta(s+z)}\right]-\frac{2 \mu^{s}\left(2 \xi^{2}-k_{2}^{2}\right) \xi}{a_{2} k_{2}^{2}}\left[\xi^{2} e^{-\alpha s-\beta z}+\alpha \beta e^{-\beta s-\alpha z}\right] \\
& \bar{\gamma}_{5}=-\frac{\alpha}{2 k_{1}^{2}} e^{-\alpha|z-s|}+\frac{\xi^{2}}{2 \beta k_{1}^{2}} e^{-\beta|z-s|}-\frac{1}{2 k_{1}^{2}} \frac{a_{1}}{a_{2}}\left[\alpha e^{-\alpha(s+z)}+\frac{\xi^{2}}{\beta} e^{-\beta(s+z)}\right]+\frac{2 \mu^{s}\left(2 \xi^{2}-k_{2}^{2}\right) \alpha \xi^{2}}{a_{2} k_{1}^{2}}\left[e^{-\alpha s-\beta z}+e^{-\beta s-\alpha z}\right],
\end{aligned}
$$

and

$$
\begin{aligned}
a_{1} & =\mu^{s}\left[\left(2 \xi^{2}-k_{2}^{2}\right)^{2}+4 \xi^{2} \alpha \beta\right], \\
a_{2} & =\mu^{s}\left[\left(2 \xi^{2}-k_{2}^{2}\right)^{2}-4 \xi^{2} \alpha \beta\right], \\
k_{1}^{2} & =\frac{\rho^{s} \omega^{2}}{\lambda^{s}+2 \mu^{s}}, \\
k_{2}^{2} & =\frac{\rho^{s} \omega^{2}}{\mu^{s},} \\
\operatorname{sgn}(z, s) & = \begin{cases}-1, & z<s, \\
1, & z>s .\end{cases}
\end{aligned}
$$

It is easy to show that the reduced results are identical to the classical half-space solution from Pooladi et al. [34], which can also be graphically confirmed by the excellent agreement between the reduced results and the half-space results as shown in Figure 2. Note that in the following computation analyses, the numerical quadrature method suggested by Pooladi et al. [34] is used to evaluate the present solution. Unless otherwise stated, the material parameters $\lambda^{s}=\mu^{s}=10 \mathrm{MPa}$ and $\rho^{s}=2500 \mathrm{~kg} \cdot \mathrm{m}^{-3}$, the load radius $r_{0}=1 \mathrm{~m}$, and the observation point coordinates $r=0, \theta=0$ are employed in the whole numerical examples.

Also, as depicted in Figure 3(a), an axisymmetric finite element model for buried vertical load case with ADINA is established to verify the present solution. In this model, the above material parameters are used; for dynamic case, the dimensionless excitation frequency $\bar{\omega}=\omega r_{0} \sqrt{\rho^{s} / \mu^{s}}=0.5$; 9node rectangle element is employed; the model length of $50 \mathrm{~m}$ has been able to eliminate the boundary effect. It should be noted that due to the software display setting for two-dimensional problems, the coordinate system of the axisymmetric FEM model is shown as the Cartesian coordinate system. The coordinate systems in the present paper and the FEM model are equivalent. It is observed from 


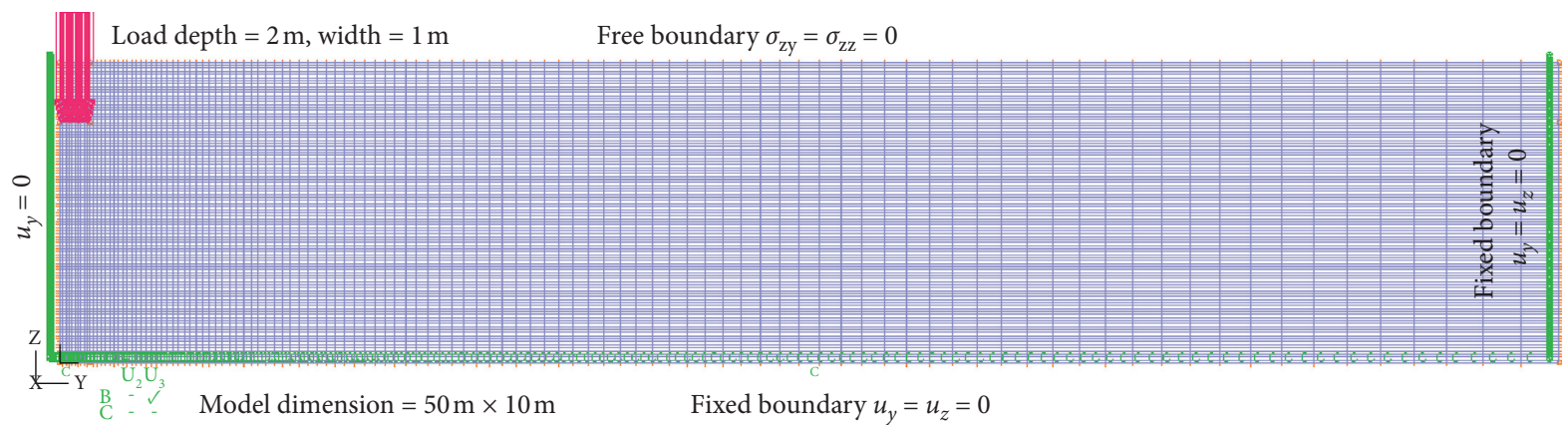

(a)

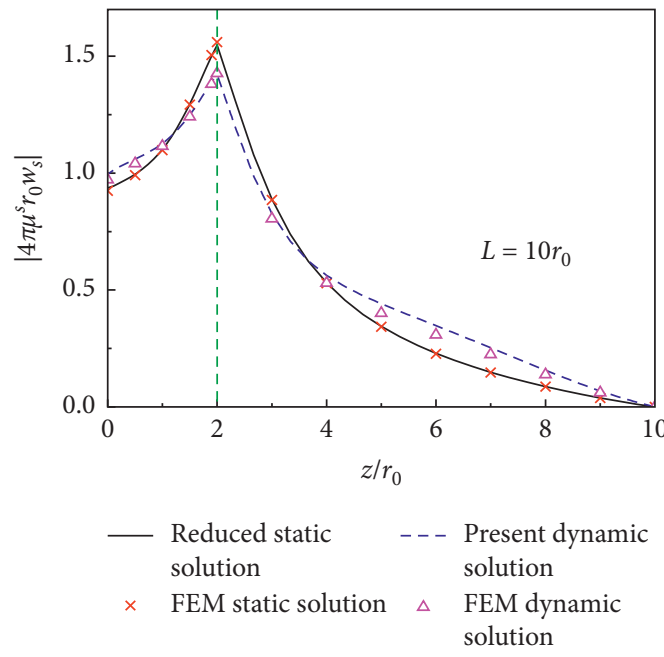

(b)

FIGURE 3: Comparisons of the present solution with the corresponding FEM solution: (a) axisymmetric finite element model by ADINA; (b) modulus of vertical displacement along with depth.

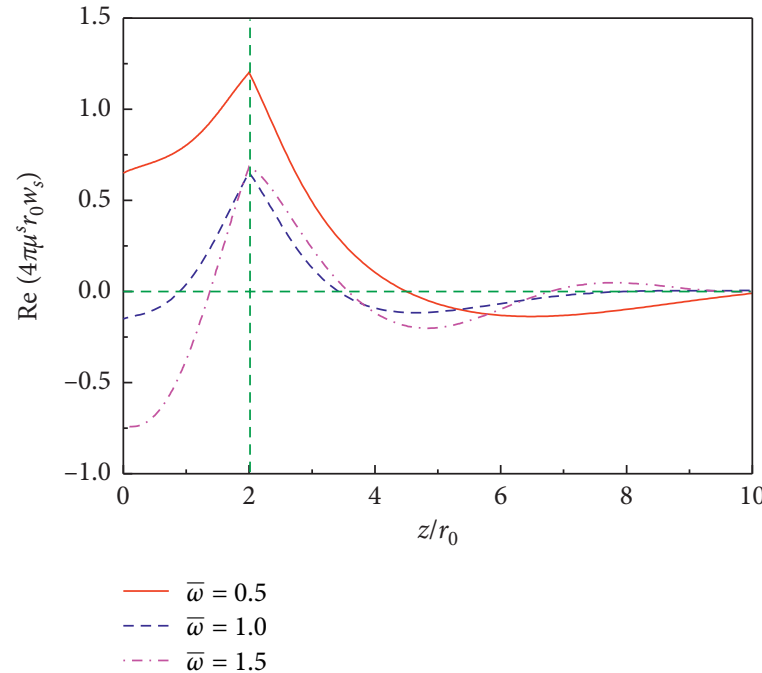

(a)

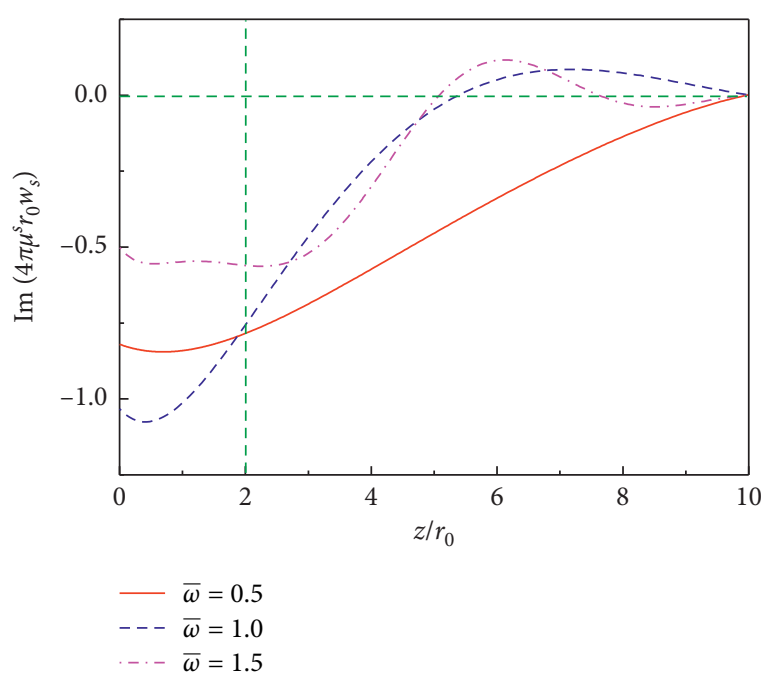

(b)

FIGURE 4: Vertical displacement $w_{s}$ induced by unit vertical load on $\pi_{s}$ : (a) real part of vertical displacement; (b) imaginary part of vertical displacement. 


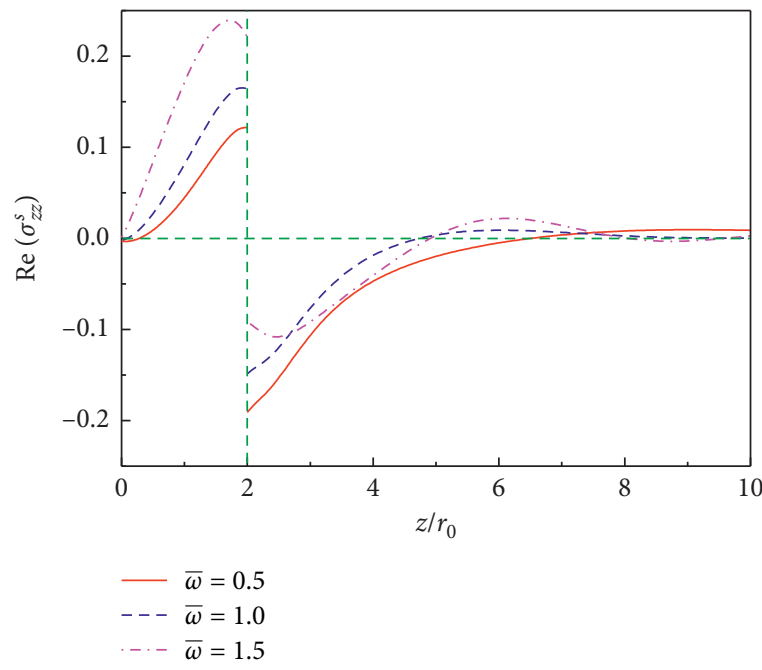

(a)

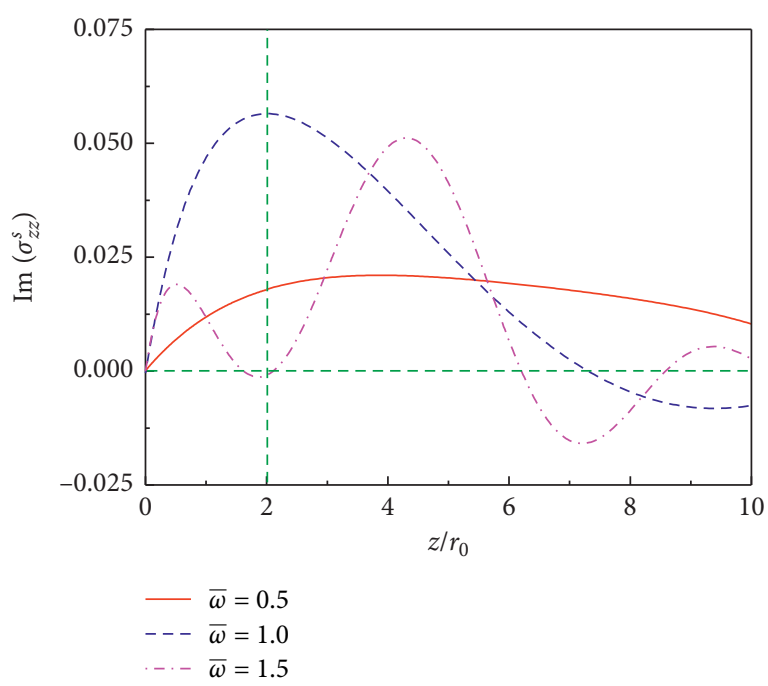

(b)

FIGURE 5: Vertical stress $\sigma_{z z}^{s}$ induced by unit vertical load on $\pi_{s}$ : (a) real part of vertical stress; (b) imaginary part of vertical stress.

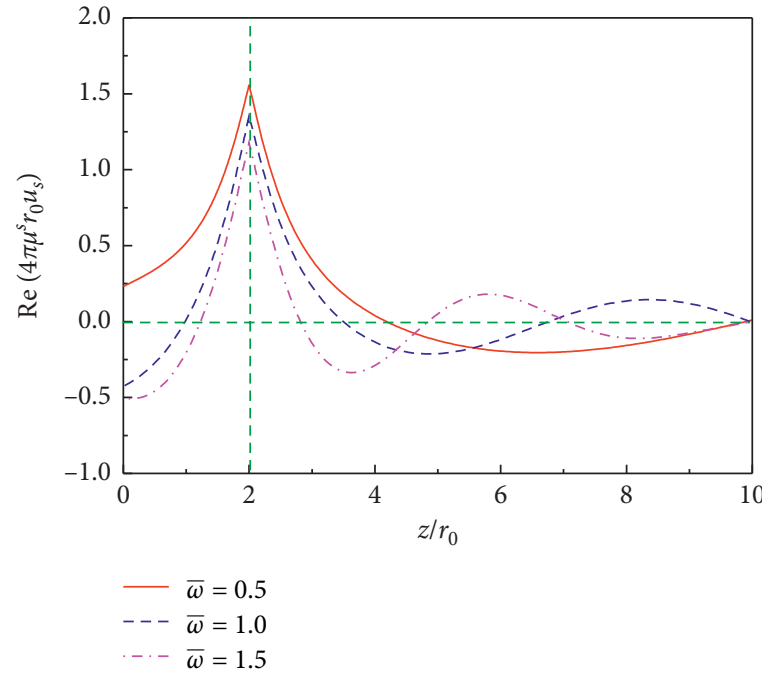

(a)

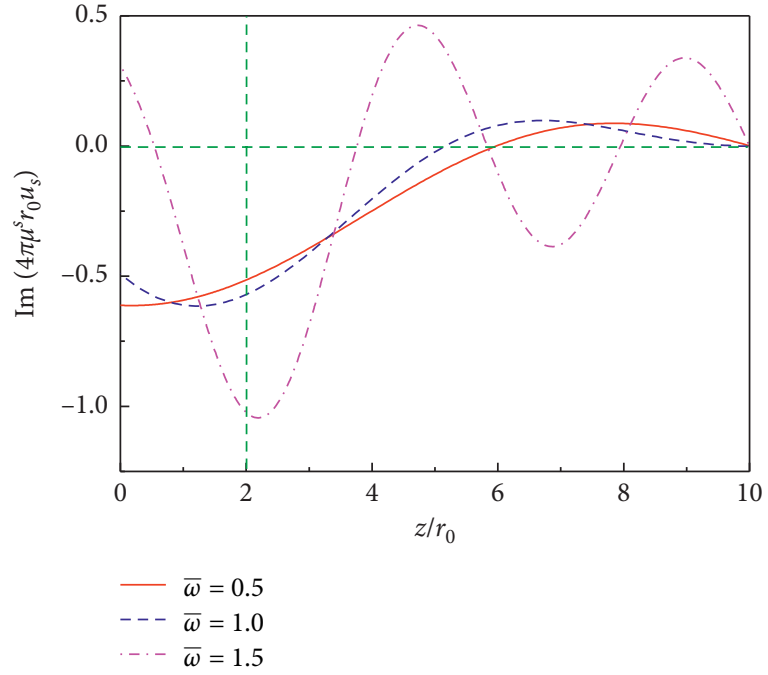

(b)

FiguRE 6: Lateral displacement $u_{s}$ induced by unit lateral load on $\pi_{s}$ : (a) real part of lateral displacement; (b) imaginary part of lateral displacement.

Figure 3(b) that these solutions agree well, and the present solution is thus validated.

Figures 4 and 5 show the displacement and stress distributions of the layer along the $z$ axis under vertical loads. From Figure 4, sharp corners are observed at the load plane $z=s$, and the displacements vanish at the soil bottom; these results are consistent with the boundary conditions (2c) and (3b). From Figure 5, the stress vanishes at the soil surface $z=0$, and the stress discontinuity is $1 / \pi$ at the load plane $z=s$; these results are consistent with the boundary conditions (3a) and (2c). With the increase of the excitation frequency, the fluctuation of the dynamic response of the layer increases. Similar behaviors can also be observed in
Figures 6 and 7 that represent lateral displacement and stress distributions of the layer along the $z$ axis under lateral loads.

The effects of the layer thickness on the vertical displacements induced by vertical loads are shown in Figures 8 and 9. Obviously, the layer thickness has significant and complicated effects on the displacements. When its thickness tends to infinity, the displacements converge to the halfspace results. This is because the waves are reflected by the rigid base and then disturb the behavior of the layer. When the layer is very thick, which means the base is located far away, fewer waves are reflected and have smaller amplitude due to attenuation, such that in this case the reflected components have negligible effects on the layer. This also 


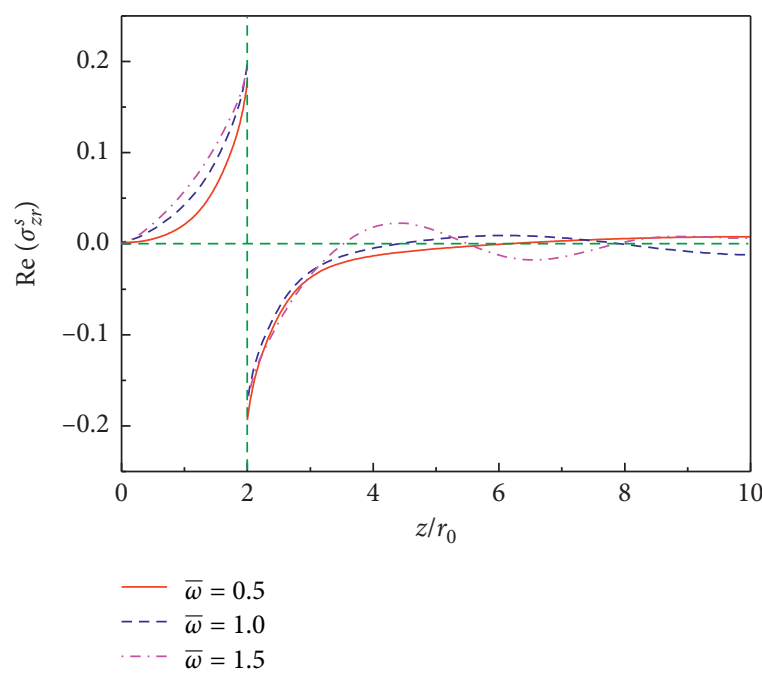

(a)

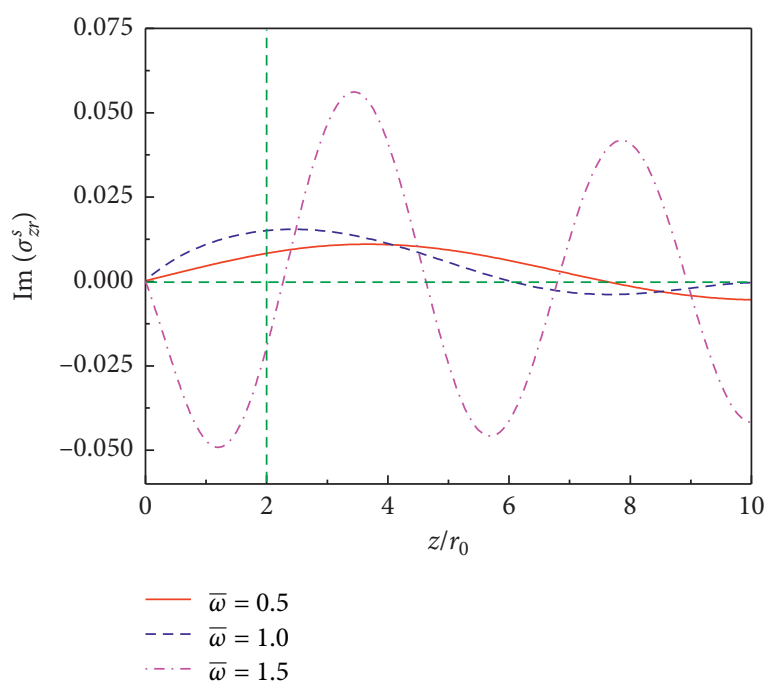

(b)

FIGURE 7: Lateral stress $\sigma_{z r}^{s}$ induced by unit lateral load on $\pi_{s}$ : (a) real part of lateral stress; (b) imaginary part of lateral stress.

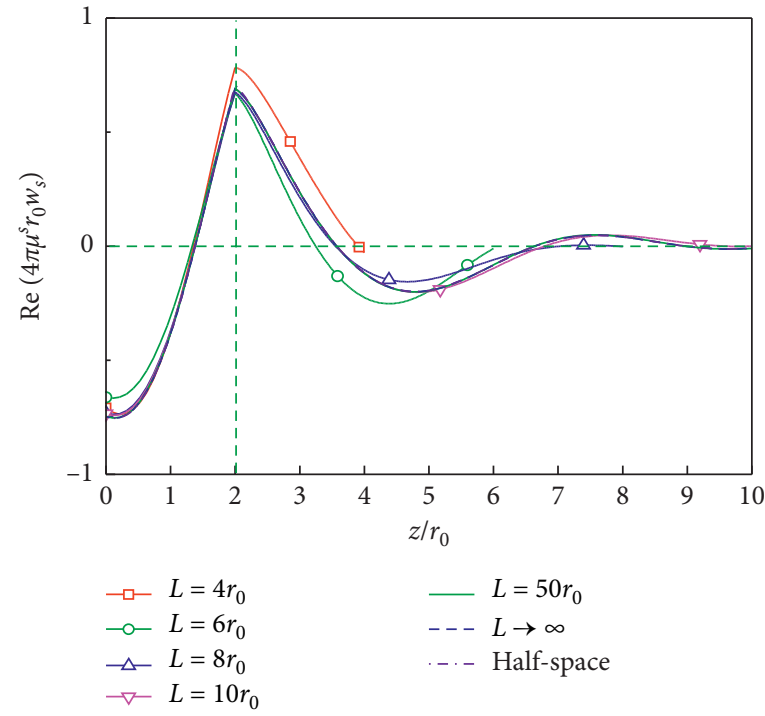

(a)

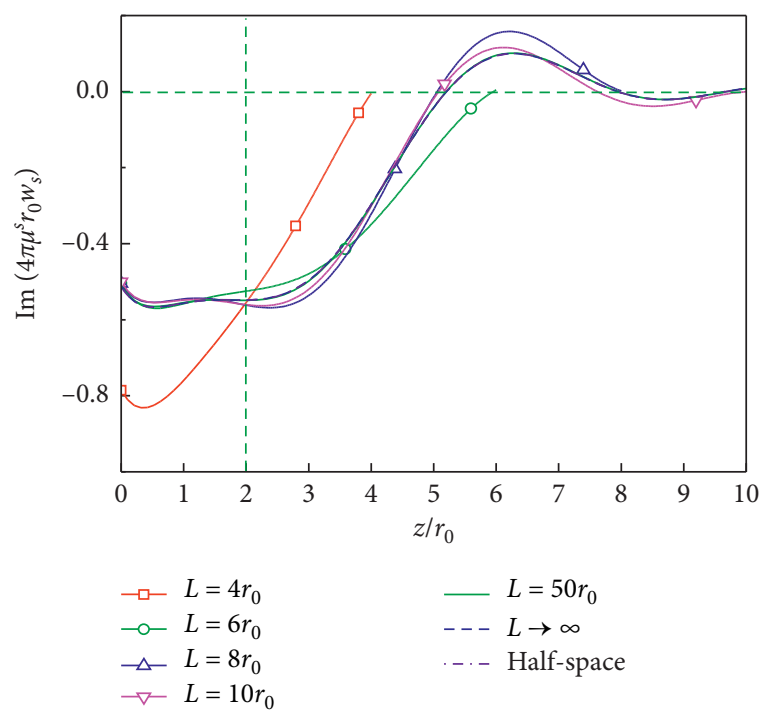

(b)

FiguRE 8: Vertical displacement $w_{s}$ induced by unit vertical load on $\pi_{s}\left(s=2 r_{0}, \bar{\omega}=1.5\right)$ : (a) real part of vertical displacement; (b) imaginary part of vertical displacement.

confirms that when the layer is thick enough (e.g., the ratio of the layer thickness beneath the load to the load radius $\geq 50$ ), it can be modeled as half-spaces for simplification.
Similar conclusions can also be drawn from Figures 10 and 11 , which represent the lateral displacements induced by lateral loads. 


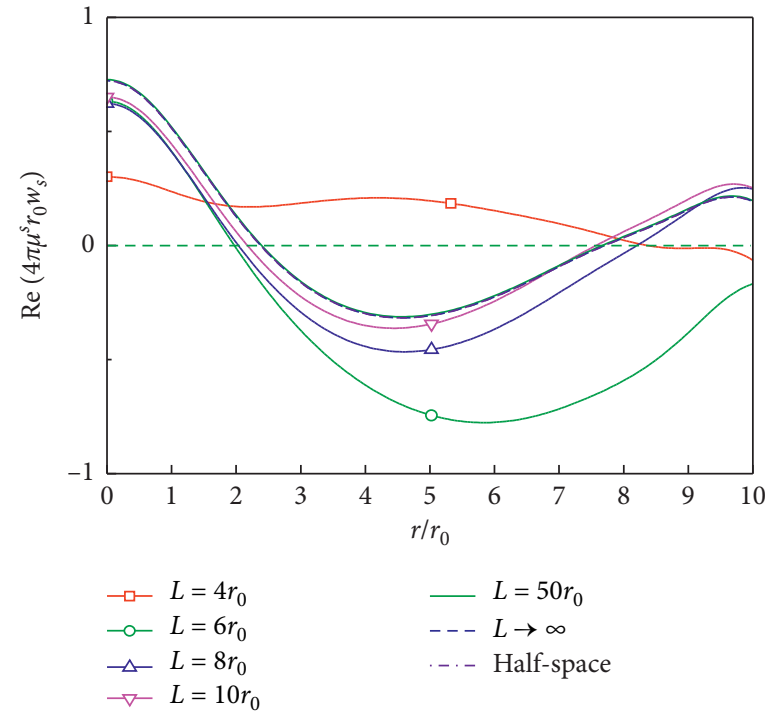

(a)

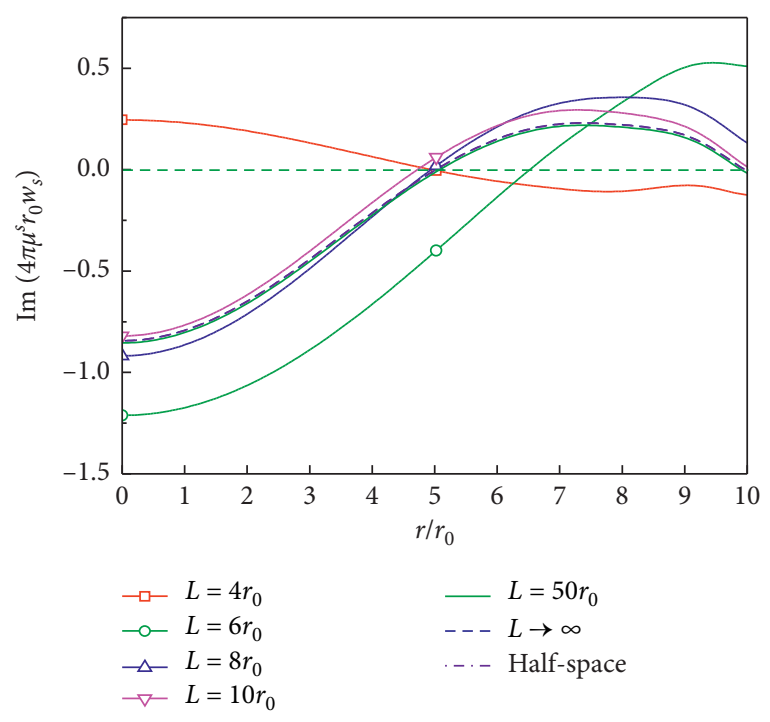

(b)

FIgURe 9: Vertical displacement $w_{s}$ at the surface $z=0$ induced by unit vertical load on $\pi_{s}\left(s=2 r_{0}, \bar{\omega}=0.5\right)$ : (a) real part of vertical displacement; (b) imaginary part of vertical displacement.

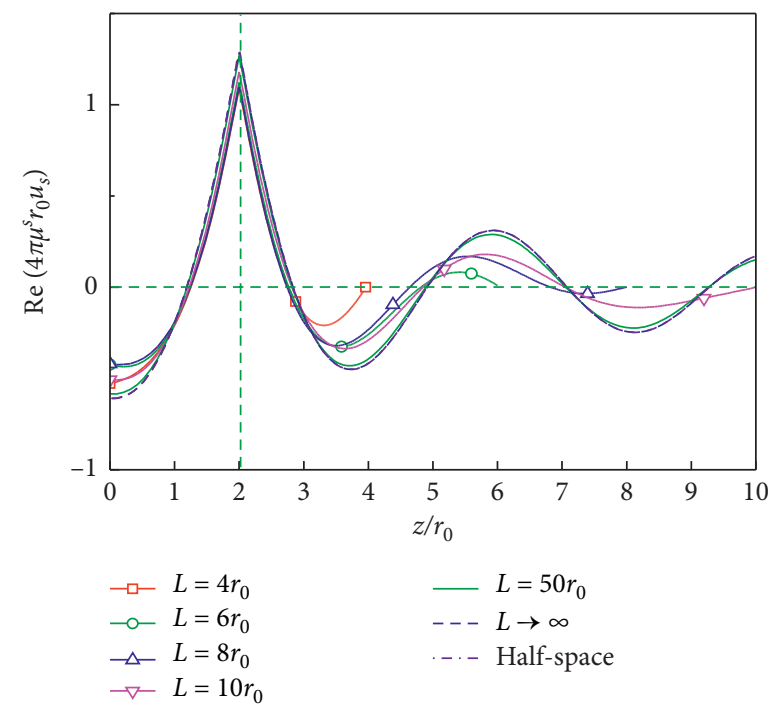

(a)

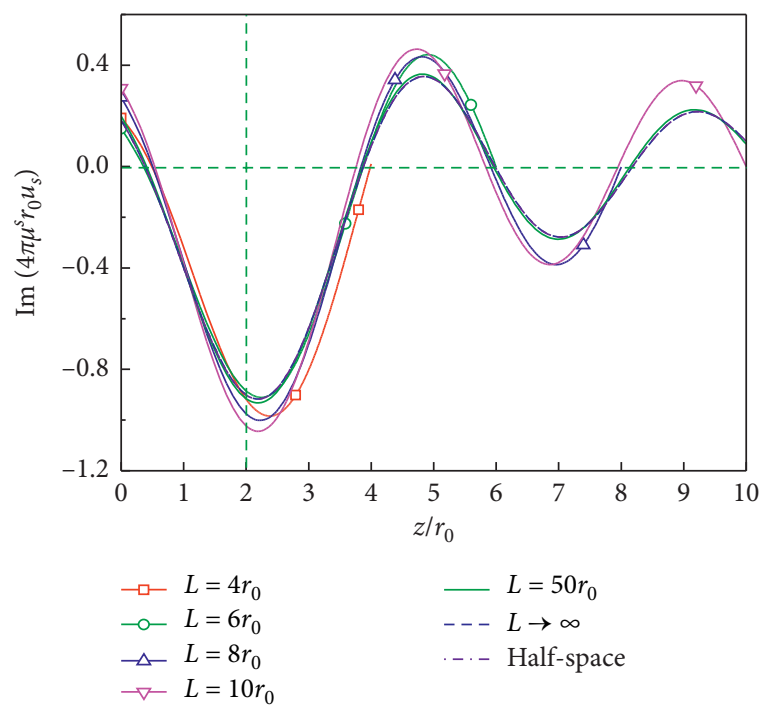

(b)

FIGURE 10: Lateral displacement $u_{s}$ induced by unit lateral load on $\pi_{s}\left(s=2 r_{0}, \bar{\omega}=1.5\right)$ : (a) real part of lateral displacement; (b) imaginary part of lateral displacement. 


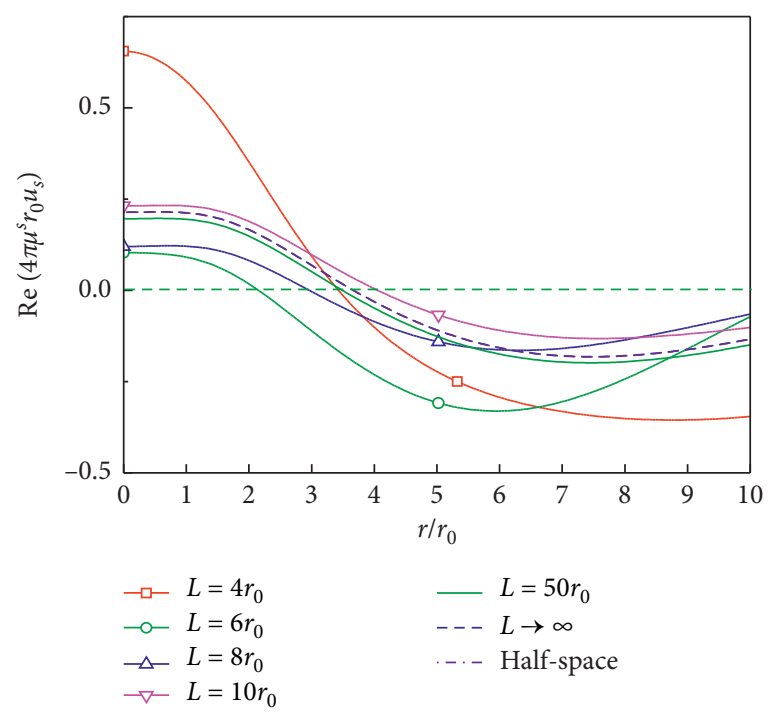

(a)

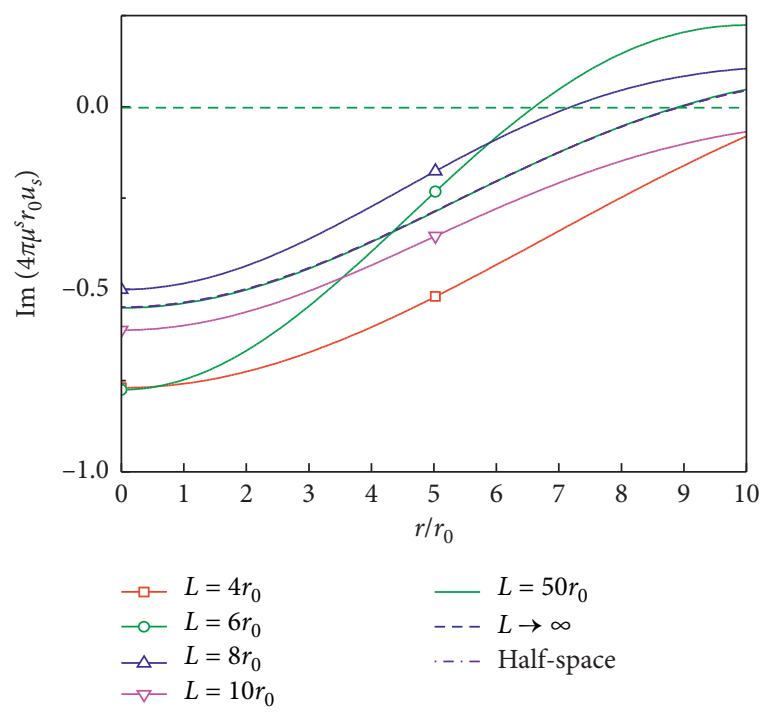

(b)

Figure 11: Lateral displacement $u_{s}$ at the surface $z=0$ induced by unit lateral load on $\pi_{s}\left(s=2 r_{0}, \bar{\omega}=0.5\right)$ : (a) real part of lateral displacement; (b) imaginary part of lateral displacement.

\section{Conclusions}

In this paper, three-dimensional general solutions of an elastic layer resting on rigid base to arbitrary, distributed, and buried loads are presented by a method of displacement potentials and integral transforms. As illustrations, results for vertical and horizontal loads of uniform circular distributions are also included. The solution is demonstrated with numerical examples and the effects of the layer thickness are examined as well. Compared with the classical results of half-spaces or surface load problems, the present solution can be used to deal with a variety of axisymmetric and asymmetric wave propagation problems in finite-layered media.

Also, it is worth noting that from the previous analysis, the generalized solutions presented in this work cover many classical results, such as half-space solutions and surface load solutions, and may have a wide range of application. By using the present solutions, other Green's functions corresponding to ring loads and point loads can be easily derived, which are of fundamental importance to analyze the problems associated with soil-structure interactions by the method of boundary integral equations.

\section{Appendix}

\section{Coefficients in Equations (19) and (20)}

The coefficients in equations (19) and (20) are listed below:

$$
\begin{aligned}
& \gamma_{1}^{-}=\frac{\xi}{2 k_{2}^{2}}\left[\xi\left(c_{1}^{-}+c_{3}^{-}\right)+\beta\left(c_{5}^{-}+c_{7}^{-}\right)\right], \\
& \gamma_{2}^{-}=\frac{1}{2 \beta\left(1+e^{-2 \beta L}\right)}\left[e^{\beta(z-s)}-e^{-2 \beta L} e^{\beta(z+s)}+e^{-\beta(z+s)}-e^{-2 \beta L} e^{-\beta(z-s)}\right], \\
& \gamma_{3}^{-}=\frac{\xi}{2 k_{1}^{2}}\left[c_{2}^{-}+c_{4}^{-}+\beta\left(c_{6}^{-}+c_{8}^{-}\right)\right], \\
& \gamma_{4}^{-}=\frac{\xi}{2 k_{2}^{2}}\left[\alpha\left(c_{1}^{-}+c_{3}^{-}\right)+\xi\left(c_{5}^{-}+c_{7}^{-}\right)\right], \\
& \gamma_{5}^{-}=\frac{1}{2 k_{1}^{2}}\left[\alpha\left(c_{2}^{-}-c_{4}^{-}\right)+\xi^{2}\left(c_{6}^{-}-c_{8}^{-}\right)\right], \\
& \gamma_{6}^{-}=\frac{\xi}{2 k_{2}^{2}}\left[\left(2 \mu^{s} \alpha^{2}-\lambda^{s} k_{1}^{2}\right)\left(c_{1}^{-}+c_{3}^{-}\right)+2 \mu^{s} \xi \beta\left(c_{5}^{-}+c_{7}^{-}\right)\right],
\end{aligned}
$$




$$
\begin{aligned}
& \gamma_{7}^{-}=\frac{1}{2 k_{1}^{2}}\left[\left(2 \mu^{s} \alpha^{2}-\lambda^{s} k_{1}^{2}\right)\left(c_{2}^{-}+c_{4}^{-}\right)+2 \mu^{s} \xi^{2} \beta\left(c_{6}^{-}+c_{8}^{-}\right)\right] \\
& \gamma_{8}^{-}=\frac{\xi}{2 k_{2}^{2}}\left[2 \xi \alpha\left(c_{1}^{-}-c_{3}^{-}\right)+\left(\beta^{2}+\xi^{2}\right)\left(c_{5}^{-}+c_{7}^{-}\right)\right] \text {, } \\
& \gamma_{9}^{-}=\frac{1}{2\left(1+e^{-2 \beta L}\right)}\left[e^{\beta(z-s)}-e^{-\beta(z+s)}-e^{-2 \beta L} e^{\beta(z+s)}+e^{-2 \beta L} e^{-\beta(z-s)}\right], \\
& \gamma_{10}^{-}=\frac{\mu^{s} \xi}{2 k_{1}^{2}}\left[2 \alpha\left(c_{2}^{-}-c_{4}^{-}\right)+\left(\beta^{2}+\xi^{2}\right)\left(c_{6}^{-}-c_{8}^{-}\right)\right] \\
& \gamma_{1}^{+}=\frac{\xi}{2 k_{2}^{2}}\left[\xi c_{1}^{+} \frac{1}{e^{\alpha L}}+\xi c_{3}^{+} \frac{1}{R}\left(\alpha \beta+\xi^{2}\right)+\beta c_{5}^{+} \frac{1}{a_{4}}\left(\alpha \beta+\xi^{2}\right) e^{-\beta L}+\beta c_{7}^{+}\right], \\
& \gamma_{2}^{+}=\frac{1}{2 \beta\left(1+e^{-2 \beta L}\right)}\left[e^{-\beta(z+s)}+e^{-\beta(z-s)}-e^{-2 \beta L} e^{\beta(z-s)}-e^{-2 \beta L} e^{\beta(z+s)}\right], \\
& \gamma_{3}^{+}=\frac{\xi}{2 k_{1}^{2}}\left[c_{2}^{+} \frac{1}{e^{\alpha L}}+c_{4}^{+} \frac{1}{R}\left(\alpha \beta+\xi^{2}\right)+\beta c_{6}^{+} \frac{1}{a_{4}}\left(\alpha \beta+\xi^{2}\right) e^{-\beta L}+\beta c_{8}^{+}\right] \text {, } \\
& \gamma_{4}^{+}=\frac{\xi}{2 k_{2}^{2}}\left[\alpha c_{1}^{+} \frac{1}{e^{\alpha L}}-\alpha c_{3}^{+} \frac{1}{R}\left(\alpha \beta+\xi^{2}\right)+\xi c_{5}^{+} \frac{1}{a_{4}}\left(\alpha \beta+\xi^{2}\right) e^{-\beta L}-\xi c_{7}^{+}\right] \\
& \gamma_{5}^{+}=\frac{1}{2 k_{1}^{2}}\left[\alpha c_{2}^{+} \frac{1}{e^{\alpha L}}-\alpha c_{4}^{+} \frac{1}{R}\left(\alpha \beta+\xi^{2}\right)+\xi^{2} c_{6}^{+} \frac{1}{a_{4}}\left(\alpha \beta+\xi^{2}\right) e^{-\beta L}-\xi^{2} c_{8}^{+}\right] \text {, } \\
& \gamma_{6}^{+}=\frac{\xi}{2 k_{2}^{2}}\left[\left(2 \mu^{s} \alpha^{2}-\lambda^{s} k_{1}^{2}\right) c_{1}^{+} \frac{1}{e^{\alpha L}}+\left(2 \mu^{s} \alpha^{2}-\lambda^{s} k_{1}^{2}\right) c_{3}^{+} \frac{1}{R}\left(\alpha \beta+\xi^{2}\right)+2 \mu^{s} \xi^{2} \beta c_{5}^{+} \frac{1}{a_{4}}\left(\alpha \beta+\xi^{2}\right) e^{-\beta L}+2 \mu^{s} \xi^{2} \beta c_{7}^{+}\right] \text {, } \\
& \gamma_{7}^{+}=\frac{1}{2 k_{1}^{2}}\left[\left(2 \mu^{s} \alpha^{2}-\lambda^{s} k_{1}^{2}\right) c_{2}^{+} \frac{1}{e^{\alpha L}}+\left(2 \mu^{s} \alpha^{2}-\lambda^{s} k_{1}^{2}\right) c_{4}^{+} \frac{1}{R}\left(\alpha \beta+\xi^{2}\right)+2 \mu^{s} \xi^{2} \beta c_{6}^{+} \frac{1}{a_{4}}\left(\alpha \beta+\xi^{2}\right) e^{-\beta L}+2 \mu^{s} \xi^{2} \beta c_{8}^{+}\right] \text {, } \\
& \gamma_{8}^{+}=\frac{\xi}{2 k_{2}^{2}}\left[2 \xi \alpha c_{1}^{+} \frac{1}{e^{\alpha L}}-2 \xi \alpha c_{3}^{+} \frac{1}{R}\left(\alpha \beta+\xi^{2}\right)+\left(\beta^{2}+\xi^{2}\right) c_{5}^{+} \frac{1}{a_{4}}\left(\alpha \beta+\xi^{2}\right) e^{-\beta L}-\left(\beta^{2}+\xi^{2}\right) c_{7}^{+}\right] \\
& \gamma_{9}^{+}=-\frac{1}{2\left(1+e^{-2 \beta L}\right)}\left[e^{-\beta(z+s)}+e^{-\beta(z-s)}+e^{-2 \beta L} e^{\beta(z-s)}+e^{-2 \beta L} e^{\beta(z+s)}\right], \\
& \gamma_{10}^{+}=\frac{\mu^{s} \xi}{2 k_{1}^{2}}\left[2 \alpha c_{2}^{+} \frac{1}{e^{\alpha L}}-2 \alpha c_{4}^{+} \frac{1}{R}\left(\alpha \beta+\xi^{2}\right)+\left(\beta^{2}+\xi^{2}\right) c_{6}^{+} \frac{1}{a_{4}}\left(\alpha \beta+\xi^{2}\right) e^{-\beta L}-\left(\beta^{2}+\xi^{2}\right) c_{8}^{+}\right] \text {, }
\end{aligned}
$$

where

$$
\begin{aligned}
& c_{1}^{-}=\left(b_{1} \frac{1}{e^{\alpha L}}-1\right) \frac{1}{\alpha} e^{\alpha(z-s)}-b_{2} \frac{1}{e^{\alpha L}} 2 \beta\left(\beta^{2}+\xi^{2}\right) e^{\alpha z-\beta s}-b_{3} \frac{1}{e^{\alpha L}} \frac{1}{\alpha} e^{\alpha(z+s)}+b_{4} \frac{1}{e^{\alpha L}} 2 \beta e^{-\beta L}\left(\beta^{2}+\xi^{2}\right) e^{\alpha z+\beta s}, \\
& c_{2}^{-}=\left(b_{1} \frac{1}{e^{\alpha L}}-1\right) e^{\alpha(z-s)}-b_{2} \frac{1}{e^{\alpha L}} 2 \xi^{2}\left(\beta^{2}+\xi^{2}\right) e^{\alpha z-\beta s}+b_{3} \frac{1}{e^{\alpha L}} e^{\alpha(z+s)}-b_{4} \frac{1}{e^{\alpha L}} 2 \xi^{2} e^{-\beta L}\left(\beta^{2}+\xi^{2}\right) e^{\alpha z+\beta s},
\end{aligned}
$$


Shock and Vibration

15

$$
\begin{aligned}
& c_{3}^{-}=\left(a_{4} a_{1}-a_{5} 2 \xi^{2} \beta e^{-\beta L} 2 \alpha\right) \frac{1}{R}\left(\alpha \beta+\xi^{2}\right) \frac{1}{\alpha} e^{-\alpha(z+s)}-\left(a_{4} 2 \mu^{s}-a_{5} e^{-\beta L}\right) \frac{1}{R}\left(\alpha \beta+\xi^{2}\right) 2 \beta\left(\beta^{2}+\xi^{2}\right) e^{-\alpha z-\beta s} \\
& -\left[\left(a_{4} a_{2}+a_{5} 2 \xi^{2} \beta e^{-\beta L} 2 \alpha\right) \frac{1}{R}\left(\alpha \beta+\xi^{2}\right)-1\right] \frac{1}{\alpha} e^{-\alpha(z-s)}+a_{5} e^{-\beta L} \frac{1}{R}\left(\alpha \beta+\xi^{2}\right) 2 \beta\left(\beta^{2}+\xi^{2}\right) e^{-\alpha z+\beta s}, \\
& c_{4}^{-}=\left(a_{4} a_{1}-a_{5} 2 \xi^{2} \beta e^{-\beta L} 2 \alpha\right) \frac{1}{R}\left(\alpha \beta+\xi^{2}\right) e^{-\alpha(z+s)}-\left(a_{4} 2 \mu^{s}-a_{5} e^{-\beta L}\right) \frac{1}{R}\left(\alpha \beta+\xi^{2}\right) 2 \xi^{2}\left(\beta^{2}+\xi^{2}\right) e^{-\alpha z-\beta s} \\
& +\left[\left(a_{4} a_{2}+a_{5} 2 \xi^{2} \beta e^{-\beta L} 2 \alpha\right) \frac{1}{R}\left(\alpha \beta+\xi^{2}\right)-1\right] e^{-\alpha(z-s)}-a_{5} e^{-\beta L} \frac{1}{R}\left(\alpha \beta+\xi^{2}\right) 2 \xi^{2}\left(\beta^{2}+\xi^{2}\right) e^{-\alpha z+\beta s}, \\
& c_{5}^{-}=\left[e^{-\beta L}-a_{3} \frac{1}{R}\left(a_{4} a_{1}-a_{5} 2 \xi^{2} \beta e^{-\beta L} 2 \alpha\right)\right] \frac{1}{a_{4}}\left(\alpha \beta+\xi^{2}\right) e^{-\beta L} 2 \xi e^{\beta z-\alpha s} \\
& -\left\{\left[e^{-\beta L}-a_{3} \frac{1}{R} 2 \alpha 2 \xi^{2} \beta\left(a_{4} 2 \mu^{s}-a_{5} e^{-\beta L}\right)\right] \frac{1}{a_{4}} e^{-\beta L}\left(\alpha \beta+\xi^{2}\right)\left(\beta^{2}+\xi^{2}\right)-1\right\} \frac{1}{\xi} e^{\beta(z-s)} \\
& +\left[e^{-\beta L}+a_{3} \frac{1}{R}\left(a_{4} a_{2}+a_{5} 2 \xi^{2} \beta e^{-\beta L} 2 \alpha\right)\right] \frac{1}{a_{4}} e^{-\beta L} 2\left(\alpha \beta+\xi^{2}\right) \xi e^{\beta z+\alpha s} \\
& -\left(1+\frac{1}{R} a_{3} a_{5} 2 \alpha 2 \xi^{2} \beta\right) \frac{1}{a_{4}}\left(\alpha \beta+\xi^{2}\right) e^{-2 \beta L}\left(\beta^{2}+\xi^{2}\right) \frac{1}{\xi} e^{\beta(z+s)}, \\
& c_{6}^{-}=\left[e^{-\beta L}-a_{3} \frac{1}{R}\left(a_{4} a_{1}-a_{5} 2 \xi^{2} \beta e^{-\beta L} 2 \alpha\right)\right] \frac{1}{a_{4}}\left(\alpha \beta+\xi^{2}\right) e^{-\beta L} 2 \alpha e^{\beta z-\alpha s} \\
& -\left\{\left[e^{-\beta L}-a_{3} \frac{1}{R} 2 \alpha 2 \xi^{2} \beta\left(a_{4} 2 \mu^{s}-a_{5} e^{-\beta L}\right)\right] \frac{1}{a_{4}}\left(\alpha \beta+\xi^{2}\right) e^{-\beta L}\left(\beta^{2}+\xi^{2}\right)-1\right\} \frac{1}{\beta} e^{\beta(z-s)} \\
& -\left[e^{-\beta L}+a_{3} \frac{1}{R}\left(a_{4} a_{2}+a_{5} 2 \xi^{2} \beta e^{-\beta L} 2 \alpha\right)\right] \frac{1}{a_{4}}\left(\alpha \beta+\xi^{2}\right) e^{-\beta L} 2 \alpha e^{\beta z+\alpha s} \\
& +\left(1+\frac{1}{R} a_{3} a_{5} 2 \alpha 2 \xi^{2} \beta\right) \frac{1}{a_{4}}\left(\alpha \beta+\xi^{2}\right) e^{-2 \beta L}\left(\beta^{2}+\xi^{2}\right) \frac{1}{\beta} e^{\beta(z+s)}, \\
& c_{7}^{-}=b_{5} 2 \xi e^{-\alpha s-\beta z}-b_{6} \frac{1}{\xi} e^{-(z+s)}-b_{7} 2 \xi e^{-\beta z+\alpha s}+\left(b_{8}-1\right) \frac{1}{\xi} e^{-\beta(z-s)}, \\
& c_{8}^{-}=b_{5} 2 \alpha e^{-\beta z-\alpha s}-b_{6} \frac{1}{\beta} e^{-\beta(z+s)}+b_{7} 2 \alpha e^{-\beta z+\alpha s}-\left(b_{8}-1\right) \frac{1}{\beta} e^{-\beta(z-s)}, \\
& c_{1}^{+}=b_{1} \frac{1}{\alpha} e^{\alpha(z-s)}-b_{2} 2 \beta\left(\beta^{2}+\xi^{2}\right) e^{\alpha z-\beta s}-b_{3} \frac{1}{\alpha} e^{\alpha(z+s)}+b_{4} 2 \beta e^{-\beta L}\left(\beta^{2}+\xi^{2}\right) e^{\alpha z+\beta s}, \\
& c_{2}^{+}=b_{1} e^{\alpha(z-s)}-b_{2} 2 \xi^{2}\left(\beta^{2}+\xi^{2}\right) e^{\alpha z-\beta s}+b_{3} e^{\alpha(z+s)}-b_{4} 2 \xi^{2} e^{-\beta L}\left(\beta^{2}+\xi^{2}\right) e^{\alpha z+\beta s}, \\
& c_{3}^{+}=\left(a_{4} a_{1}-a_{5} 2 \xi^{2} \beta e^{-\beta L} 2 \alpha\right) \frac{1}{\alpha} e^{-\alpha(z+s)}-\left(a_{4} 2 \mu^{s}-a_{5} e^{-\beta L}\right)\left(\beta^{2}+\xi^{2}\right) 2 \beta e^{-\alpha z-\beta s} \\
& -\left(a_{4} a_{2}+a_{5} 2 \xi^{2} \beta e^{-\beta L} 2 \alpha\right) \frac{1}{\alpha} e^{-\alpha(z-s)}+a_{5} e^{-\beta L} 2 \beta\left(\beta^{2}+\xi^{2}\right) e^{-\alpha z+\beta s} \text {, } \\
& c_{4}^{+}=\left(a_{4} a_{1}-a_{5} 2 \xi^{2} \beta e^{-\beta L} 2 \alpha\right) e^{-\alpha(z+s)}-\left(a_{4} 2 \mu^{s}-a_{5} e^{-\beta L}\right) 2 \xi^{2}\left(\beta^{2}+\xi^{2}\right) e^{-\alpha z-\beta s} \\
& +\left(a_{4} a_{2}+a_{5} 2 \xi^{2} \beta e^{-\beta L} 2 \alpha\right) e^{-\alpha(z-s)}-a_{5} e^{-\beta L} 2 \xi^{2}\left(\beta^{2}+\xi^{2}\right) e^{-\alpha z+\beta s}, \\
& c_{5}^{+}=\left[e^{-\beta L}-a_{3} \frac{1}{R}\left(a_{4} a_{1}-a_{5} 2 \xi^{2} \beta e^{-\beta L} 2 \alpha\right)\right] 2 \xi e^{\beta z-\alpha s}-\left[e^{-\beta L}-a_{3} \frac{1}{R} 2 \alpha 2 \xi^{2} \beta\left(a_{4} 2 \mu^{s}-a_{5} e^{-\beta L}\right)\right]\left(\beta^{2}+\xi^{2}\right) \frac{1}{\xi} e^{\beta(z-s)} \\
& +\left[e^{-\beta L}+a_{3} \frac{1}{R}\left(a_{4} a_{2}+a_{5} 2 \xi^{2} \beta e^{-\beta L} 2 \alpha\right)\right] 2 \xi e^{\beta z+\alpha s}-\left(1+\frac{1}{R} a_{3} a_{5} 2 \alpha 2 \xi^{2} \beta\right) e^{-\beta L}\left(\beta^{2}+\xi^{2}\right) \frac{1}{\xi} e^{\beta(z+s)},
\end{aligned}
$$




$$
\begin{aligned}
& c_{6}^{+}=\left[e^{-\beta L}-a_{3} \frac{1}{R}\left(a_{4} a_{1}-a_{5} 2 \xi^{2} \beta e^{-\beta L} 2 \alpha\right)\right] 2 \alpha e^{\beta z-\alpha s}-\left[e^{-\beta L}-a_{3} \frac{1}{R} 2 \alpha 2 \xi^{2} \beta\left(a_{4} 2 \mu^{s}-a_{5} e^{-\beta L}\right)\right]\left(\beta^{2}+\xi^{2}\right) \frac{1}{\beta} e^{\beta(z-s)} \\
& -\left[e^{-\beta L}+a_{3} \frac{1}{R}\left(a_{4} a_{2}+a_{5} 2 \xi^{2} \beta e^{-\beta L} 2 \alpha\right)\right] 2 \alpha e^{\beta z+\alpha s}+\left(1+\frac{1}{R} a_{3} a_{5} 2 \alpha 2 \xi^{2} \beta\right) e^{-\beta L}\left(\beta^{2}+\xi^{2}\right) \frac{1}{\beta} e^{\beta(z+s)}, \\
& c_{7}^{+}=b_{5} 2 \xi e^{-\beta z-\alpha s}-b_{6} \frac{1}{\xi} e^{-\beta(z+s)}-b_{7} 2 \xi e^{-\beta z+\alpha s}+b_{8} \frac{1}{\xi} e^{-\beta(z-s)}, \\
& c_{8}^{+}=b_{5} 2 \alpha e^{-\beta z-\alpha s}-b_{6} \frac{1}{\beta} e^{-\beta(z+s)}+b_{7} 2 \alpha e^{-\beta z+\alpha s}-b_{8} \frac{1}{\beta} e^{-\beta(z-s)}, \\
& b_{1}=\left(\alpha \beta-\xi^{2}\right)\left(a_{4} a_{1}-a_{5} 2 \xi^{2} \beta e^{-\beta L} 2 \alpha\right) \frac{1}{R} e^{-\alpha L}-2 \xi^{2} \beta 2 \alpha \frac{1}{a_{4}}\left[e^{-\beta L}-a_{3} \frac{1}{R}\left(a_{4} a_{1}-a_{5} 2 \xi^{2} \beta e^{-\beta L} 2 \alpha\right)\right], \\
& b_{2}=\left(\alpha \beta-\xi^{2}\right) e^{-\alpha L}\left(a_{4} 2 \mu^{s}-a_{5} e^{-\beta L}\right) \frac{1}{R}-\left[e^{-\beta L}-a_{3} \frac{1}{R} 2 \alpha 2 \xi^{2} \beta\left(a_{4} 2 \mu^{s}-a_{5} e^{-\beta L}\right)\right] \frac{1}{a_{4}}, \\
& b_{3}=\left(\alpha \beta-\xi^{2}\right) e^{-\alpha L}\left(a_{4} a_{2}+a_{5} 2 \xi^{2} \beta e^{-\beta L} 2 \alpha\right) \frac{1}{R}+2 \xi^{2} \beta\left[e^{-\beta L}+a_{3} \frac{1}{R}\left(a_{4} a_{2}+a_{5} 2 \xi^{2} \beta e^{-\beta L} 2 \alpha\right)\right] \frac{1}{a_{4}} 2 \alpha, \\
& b_{4}=\left(\alpha \beta-\xi^{2}\right) e^{-\alpha L} a_{5} \frac{1}{R}+\left(1+\frac{1}{R} a_{3} a_{5} 2 \alpha 2 \xi^{2} \beta\right) \frac{1}{a_{4}}, \\
& b_{5}=\frac{1}{\left(\beta^{2}+\xi^{2}\right)} b_{1} \frac{1}{e^{\alpha L}}-\frac{1}{\left(\beta^{2}+\xi^{2}\right)}\left(a_{4} a_{1}-a_{5} 2 \xi^{2} \beta e^{-\beta L} 2 \alpha\right) \frac{1}{R}\left(\alpha \beta+\xi^{2}\right) \\
& +\left[e^{-\beta L}-a_{3} \frac{1}{R}\left(a_{4} a_{1}-a_{5} 2 \xi^{2} \beta e^{-\beta L} 2 \alpha\right)\right] \frac{1}{a_{4}}\left(\alpha \beta+\xi^{2}\right) e^{-\beta L}-\frac{1}{\left(\beta^{2}+\xi^{2}\right)}, \\
& b_{6}=2 \alpha b_{2} \frac{1}{e^{\alpha L}} 2 \xi^{2} \beta-2 \alpha\left(a_{4} 2 \mu^{s}-a_{5} e^{-\beta L}\right) \frac{1}{R}\left(\alpha \beta+\xi^{2}\right) 2 \xi^{2} \beta \\
& +\left[e^{-\beta L}-a_{3} \frac{1}{R} 2 \alpha 2 \xi^{2} \beta\left(a_{4} 2 \mu^{s}-a_{5} e^{-\beta L}\right)\right] \frac{1}{a_{4}} e^{-\beta L}\left(\alpha \beta+\xi^{2}\right)\left(\beta^{2}+\xi^{2}\right)-1, \\
& b_{7}=\frac{1}{\left(\beta^{2}+\xi^{2}\right)} b_{3} \frac{1}{e^{\alpha L}}-\frac{1}{\left(\beta^{2}+\xi^{2}\right)}\left(a_{4} a_{2}+a_{5} 2 \xi^{2} \beta e^{-\beta L} 2 \alpha\right) \frac{1}{R}\left(\alpha \beta+\xi^{2}\right) \\
& -\left[e^{-\beta L}+a_{3} \frac{1}{R}\left(a_{4} a_{2}+a_{5} 2 \xi^{2} \beta e^{-\beta L} 2 \alpha\right)\right] \frac{1}{a_{4}} e^{-\beta L}\left(\alpha \beta+\xi^{2}\right)+\frac{1}{\left(\beta^{2}+\xi^{2}\right)}, \\
& b_{8}=2 \alpha b_{4} \frac{1}{e^{\alpha L}} 2 \xi^{2} \beta e^{-\beta L}-2 \alpha a_{5} e^{-\beta L} \frac{1}{R}\left(\alpha \beta+\xi^{2}\right) 2 \xi^{2} \beta-\left(1+\frac{1}{R} a_{3} a_{5} 2 \alpha 2 \xi^{2} \beta\right) \frac{1}{a_{4}}\left(\alpha \beta+\xi^{2}\right) e^{-2 \beta L}\left(\beta^{2}+\xi^{2}\right)+1 \\
& R=\left[a_{2}\left(\alpha \beta+\xi^{2}\right)+a_{1}\left(\alpha \beta-\xi^{2}\right) e^{-2 \alpha L}\right] a_{4}-4 \xi^{2} \alpha \beta a_{3} a_{5} \\
& a_{1}=\left(\beta^{2}+\xi^{2}\right)\left(2 \mu^{s} \alpha^{2}-\lambda^{s} k_{1}^{2}\right)+4 \mu^{s} \xi^{2} \alpha \beta \\
& a_{2}=\left(\beta^{2}+\xi^{2}\right)\left(2 \mu^{s} \alpha^{2}-\lambda^{s} k_{1}^{2}\right)-4 \mu^{s} \xi^{2} \alpha \beta \\
& a_{3}=\left(\beta^{2}+\xi^{2}\right) e^{-\alpha L}-\left(\alpha \beta+\xi^{2}\right) e^{-\beta L}+\left(\alpha \beta-\xi^{2}\right) e^{-(2 \alpha+\beta) L} \\
& a_{4}=\left(\beta^{2}+\xi^{2}\right)\left(\alpha \beta-\xi^{2}\right)+\left(\beta^{2}+\xi^{2}\right)\left(\alpha \beta+\xi^{2}\right) e^{-2 \beta L}-4 \alpha \beta \xi^{2} e^{-(\alpha+\beta) L}, \\
& a_{5}=2 \mu^{s}\left(\beta^{2}+\xi^{2}\right)\left(\alpha \beta+\xi^{2}\right) e^{-\beta L}-a_{1} e^{-\alpha L},
\end{aligned}
$$


and

$$
\begin{aligned}
X_{n} & =\widetilde{P}_{n}^{n-1}(\xi)-i \widetilde{Q}_{n}^{n-1}(\xi), \\
Y_{n} & =\widetilde{P}_{n}^{n+1}(\xi)-i \widetilde{Q}_{n}^{n+1}(\xi), \\
Z_{n} & =\widetilde{R}_{n}^{n}(\xi) .
\end{aligned}
$$

\section{Data Availability}

The data used to support the findings of this study are available from the corresponding author upon request.

\section{Conflicts of Interest}

The authors declare that they have no conflicts of interest.

\section{Acknowledgments}

This study was supported by the National Natural Science Foundation of China (grant nos. 51908070, 51978085, 51911530215 , and 51927814), the Natural Science Foundation of Hunan Province (2020JJ5596), the Open Funds of the National Engineering Laboratory of Highway Maintenance Technology (grant no. kfj190103), and the Key Laboratory of Road Structure and Material of the Ministry of Transport (grant no. kfj170304) (Changsha University of Science \& Technology).

\section{References}

[1] Z. N. Ba, J. W. Liang, V. W. Lee, and H. Ji, "3D dynamic response of a multi-layered transversely isotropic half-space subjected to a moving point load along a horizontal straight line with constant speed," International Journal of Solids and Structures, vol. 100-101, pp. 427-445, 2016.

[2] Z. N. Ba, Z. Q. Kang, and V. W. Lee, "Plane strain dynamic responses of a multi-layered transversely isotropic saturated half-space," International Journal of Engineering Science, vol. 119, pp. 55-77, 2017.

[3] N. Zhang, Y. F. Gao, Y. X. Wu, and F. Zhang, "A note on nearfield site amplification effects of ground motion from a radially inhomogeneous valley," Earthquake Engineering and Engineering Vibration, vol. 17, no. 4, pp. 707-718, 2018.

[4] H. Lamb, "On the propagation tremors over the surface of an elastic solid," Philosophical Transactions of the Royal Society of London, Series A, vol. 203, no. 359-371, pp. 1-42, 1904.

[5] L. R. Johnson, "Green's function for Lamb's problem," Geophysical Journal International, vol. 37, no. 1, pp. 99-131, 1974.

[6] Y.S. R. Pak, "Asymmetric wave propagation in an elastic halfspace by a method of potentials," Journal of Applied Mechanics, vol. 54, no. 1, pp. 121-126, 1987.

[7] M. A. Biot, "Theory of propagation of elastic waves in a fluidsaturated porous solid. I. Low-Frequency range," Journal of the Acoustical Society of America, vol. 28, no. 2, pp. 168-178, 1956.

[8] H. S. Lee, "Viscowave-a new solution for viscoelastic wave propagation of layered structures subjected to an impact load," International Journal of Pavement Engineering, vol. 15, no. 6, pp. 542-557, 2014.

[9] K. Z. Yan, H. B. Xu, and L. Y. You, "Analytical layer-element approach for wave propagation of transversely isotropic pavement," International Journal of Pavement Engineering, vol. 17, no. 3, pp. 275-282, 2016.

[10] S. Jones, "Harmonic response of layered half-space using reduced finite element model with perfectly-matched layer boundaries," Soil Dynamics and Earthquake Engineering, vol. 92, pp. 1-8, 2017.

[11] H. Liu and E. N. Pan, "Time-harmonic loading over transversely isotropic and layered elastic half-spaces with imperfect interfaces," Soil Dynamics and Earthquake Engineering, vol. 107, pp. 35-47, 2018.

[12] S. Andersen, E. Levenberg, and M. B. Andersen, "Efficient reevaluation of surface displacements in a layered elastic halfspace," International Journal of Pavement Engineering, vol. 21, no. 4, pp. 1-8, 2018.

[13] L. Y. You, K. Z. Yan, and N. Y. Liu, "Assessing the mechanical responses for anisotropic multi-layered medium under harmonic moving load by Spectral Element Method (SEM)," Applied Mathematical Modelling, vol. 67, pp. 22-37, 2019.

[14] S. Paul, "On the displacements produced in a porous elastic half-space by an impulsive line load. (Non-dissipative case)," Pure and Applied Geophysics PAGEOPH, vol. 114, no. 4, pp. 605-614, 1976a.

[15] S. Paul, "On the disturbance produced in a semi-infinite poroelastic medium by a surface load," Pure and Applied Geophysics, vol. 114, no. 4, pp. 615-627, 1976 b.

[16] M. R. Halpern and P. Christiano, "Response of poroelastic half-space to steady-state harmonic surface tractions," International Journal for Numerical and Analytical Methods in Geomechanics, vol. 10, no. 6, pp. 609-632, 1986.

[17] U. G. A. Puswewala and R. K. N. D. Rajapakse, "Axisymmetric fundamental solutions for a completely saturated porous elastic solid," International Journal of Engineering Science, vol. 26, no. 5, pp. 419-436, 1988.

[18] T. Senjuntichai and R. K. N. D. Rajapakse, "Dynamic Green's functions of homogeneous poroelastic half-plane," Journal of Engineering Mechanics, vol. 120, no. 11, pp. 2381-2404, 1994.

[19] B. Jin and H. Liu, "Dynamic response of a poroelastic halfspace to tangential surface loading," Mechanics Research Communications, vol. 28, no. 1, pp. 63-70, 2001b.

[20] S. J. Feng, Y. C. Li, H. X. Chen, and Z. L. Chen, "Response of pavement and stratified ground due to vehicle loads considering rise of water table," International Journal of Pavement Engineering, vol. 20, no. 2, pp. 191-203, 2018.

[21] A. Khojasteh, M. Rahimian, and R. Y. S. Pak, "Asymmetric dynamic Green's functions in a two-Layered transversely isotropic half-Space," Journal of Engineering Mechanics, vol. 134, no. 9, pp. 777-787, 2008.

[22] J. Liu, P. C. Zhang, and G. Lin, "Elastodynamic response for the multi-layered transversely isotropic piezoelectric solid subjected to time-harmonic loads," Composite Structures, vol. 153, pp. 755-772, 2016.

[23] G. Lin, Z. J. Han, S. Lu, and J. Liu, "Wave motion equation and the dynamic Green's function for a transverse isotropic multilayered half-space," Soils and Foundations, vol. 57, pp. 397-411, 2017.

[24] J. Park and A. M. Kaynia, "Stiffness matrices for fluid and anisotropic soil layers with applications in soil dynamics," Soil Dynamics and Earthquake Engineering, vol. 115, pp. 169-182, 2018.

[25] B. Noori, R. Arcos, A. Clot, and J. Romeu, "A method based on 3D stiffness matrices in Cartesian coordinates for computation of 2.5D elastodynamic Green's functions of layered half-spaces," Soil Dynamics and Earthquake Engineering, vol. 114, pp. 154-158, 2018. 
[26] Z. Y. Ai and Z. X. Li, "Time-harmonic response of transversely isotropic multilayered half-space in a cylindrical coordinate system," Soil Dynamics and Earthquake Engineering, vol. 66, pp. 69-77, 2014.

[27] Z. Y. Ai, J. J. Yang, and H. T. Li, "General solutions of transversely isotropic multilayered media subjected to rectangular time-harmonic or moving loads," Applied Mathematical Modelling, vol. 75, pp. 865-891, 2019.

[28] A. J. Philippacopoulos, "Buried point source in a poroelastic half-space," Journal of Engineering Mechanics, vol. 123, no. 8, pp. 860-869, 1997.

[29] B. Jin and H. Liu, "Dynamic response of a poroelastic halfspace to horizontal buried loading," International Journal of Solids and Structures, vol. 38, no. 44-45, pp. 8053-8064, 2001a.

[30] X. L. Zhou, J. H. Wang, and J. F. Lu, "Transient foundation solution of saturated soil to impulsive concentrated loading," Soil Dynamics and Earthquake Engineering, vol. 22, no. 4, pp. 273-281, 2002.

[31] S. L. Chen, L. Z. Chen, and E. Pan, "Three-dimensional timeharmonic Green's functions of saturated soil under buried loading," Soil Dynamics and Earthquake Engineering, vol. 27, no. 5, pp. 448-462, 2007.

[32] P. Zheng, S. X. Zhao, and D. Ding, "Dynamic Green's functions for a poroelastic half-space," Acta Mechanica, vol. 224, no. 1, pp. 17-39, 2013.

[33] P. Zheng, B. Y. Ding, and S. X. Zhao, "Frequency domain fundamental solutions for a poroelastic half-space," Acta Mechanica Sinica, vol. 30, no. 2, pp. 206-213, 2014.

[34] A. Pooladi, M. Rahimian, and Y. S. R. Pak, "Three-dimensional time-harmonic fundamental solutions for a fluid-saturated poroelastic half-space with partially permeable free surface," International Journal for Numerical and Analytical Methods in Geomechanics, vol. 40, no. 14, pp. 1903-1935, 2016.

[35] J. L. Zheng, "Dynamic response of elastic layer on stiff foundation under time harmonic surface vertical concentrated load," Applied Mathematics and Mechanics-English Edition, vol. 8, no. 10, pp. 919-927, 1987.

[36] S. L. Chen, L. M. Zhang, and L. Z. Chen, "Consolidation of a finite transversely isotropic soil layer on a rough impervious base," Journal of Engineering Mechanics, vol. 131, no. 12, pp. 1279-1290, 2005.

[37] J. Kim, "General viscoelastic solutions for multilayered systems subjected to static and moving loads," Journal of Materials in Civil Engineering, vol. 23, no. 7, pp. 1007-1016, 2011.

[38] H. C. Dan, L. H. He, and L. H. Zhao, "Coupled hydro-mechanical response of saturated asphalt pavement under moving traffic load," International Journal of Pavement Engineering, vol. 16, no. 2, pp. 125-143, 2015.

[39] W. K. Yuan and G. F. Wang, "Cylindrical indentation of an elastic bonded layer with surface tension," Applied Mathematical Modelling, vol. 65, pp. 597-613, 2019. 